
\title{
25 Research Sourere \\ Intrinsic Disorder in Proteins Associated With Oxidative Stress-Induced JNK Signaling
}

Bhuvaneshwari R. Gehi

Indian Institute of Technology Mandi

Kundlik Gadhave

Indian Institute of Technology Mandi

Vladimir N. Uversky ( $\nabla$ vuversky@usf.edu )

University of South Florida https://orcid.org/0000-0002-4037-5857

\section{Rajanish Giri}

Indian Institute of Technology Mandi

\section{Research Article}

Keywords: c-Jun N-terminal kinase (JNK) signaling pathway, Oxidative stress, Intrinsically disordered proteins, Intrinsically disordered protein regions, Molecular recognition features, Posttranslational modifications, Short linear Motifs

Posted Date: November 16th, 2021

DOI: https://doi.org/10.21203/rs.3.rs-1048458/v1

License: (c) (i) This work is licensed under a Creative Commons Attribution 4.0 International License. Read Full License

Version of Record: A version of this preprint was published at Cellular and Molecular Life Sciences on March 24th, 2022. See the published version at https://doi.org/10.1007/s00018-022-04230-4. 


\section{Abstract}

The c-Jun N-terminal kinase (JNK) signaling cascade is a mitogen-activated protein kinase (MAPK) signaling pathway that can be activated in response to a wide range of environmental stimuli. Based on the type, degree, and duration of the stimulus, the JNK signaling cascade dictates the fate of the cell by influencing gene expression through its substrate transcription factors. Oxidative stress is a result of a disturbance in the pro-oxidant/anti-oxidant homeostasis of the cell and is associated with a large number of diseases, such as neurodegenerative disorders, cancer, diabetes, cardiovascular diseases, and disorders of the immune system, where it activates the JNK signaling pathway. Among different biological roles ascribed to the intrinsically disordered proteins (IDPs) and hybrid proteins containing ordered domains and intrinsically disordered protein regions (IDPRs) are signaling hub functions, as intrinsic disorder allows proteins to undertake multiple interactions, each with a different consequence. In order to ensure precise signaling, cellular abundance of IDPs is highly regulated, and mutations or changes in abundance of IDPs/IDPRs are often associated with disease. In this study, we have used a combination of six disorder predictors to evaluate the presence of intrinsic disorder in proteins associated with the oxidative stress-induced JNK signaling cascade, and as per our findings, none of the 18 proteins involved in this pathway are ordered. Highest level of intrinsic disorder was observed in the scaffold proteins, JIP1, JIP2, JIP3; dual specificity phosphatases, MKP5, MKP7; a phospho-serine binding protein 14-3-3ろ, and transcription factor c-Jun. The MAP3Ks, MAP2Ks, MAPKs, TRAFs, and thioredoxin were the proteins that were predicted to be moderately disordered. Furthermore, to characterize the predicted IDPs/IDPRs in the proteins of the JNK signaling cascade, we identified the molecular recognition features (MoRFs), posttranslational modification (PTM), sites and short linear motifs (SLiMs) associated with the disordered regions. These findings will serve as a foundation for experimental characterization of disordered regions in these proteins, which represents a crucial step for better understanding of the roles of IDPRs in diseases associated with this important pathway.

\section{Introduction}

In order to ensure survival, cells have to adapt to a wide range of environmental stimuli. These stimuli could be of a physical nature, such as heat, UV radiation, osmotic stress, pH variation, oxidative stress, mechanical stress, or of a chemical nature, such as stimuli generated by cytokines, hormones, growth factors, amount of nutrients, and other environmental stresses [1]. Cells have implemented a wide range of complex signaling pathways that co-operate to mitigate the adverse effects that could result from these stimuli. Among these pathways, the Mitogen activated protein kinase (MAPK) signaling pathways relay, amplify, and integrate extracellular signals, and thereby control the cell's physiological response to these conditions [2].

The c-Jun N-terminal kinases (JNKs) belong to the sub-family of Mitogen activated protein kinases (MAPKs). This kinase sub-family also includes p38 MAPKs and Extracellular signal-regulated kinases (ERKs) [3]. The JNK group of MAPKs are multi-functional proteins that have been implicated in regulation of important physiological processes, including embryonic development, neuronal development and 
regeneration, immune responses, cytoskeletal protein dynamics, cell proliferation and apoptosis. The JNKs are also known as Stress activated protein kinases (SAPKs), because the JNK signaling pathway is activated mainly in response to biotic and abiotic stresses and mediates cell responses to such conditions $[4,5]$. JNKs were originally identified for their ability to phosphorylate the transcription factor cJun in response to stress conditions and enable it to transactivate the expression of stress related genes $[1,3,6]$.

Similar to other MAPK pathways, JNK signaling is also "three-tiered". The top tier consists of Mitogen activated protein kinase kinase kinases (MAP3Ks) [4]. These MAP3Ks can promote apoptosis via JNK signaling in a cell type- and stimuli-specific manner. At least 20 different MAP3Ks have been identified, out of which 14 are known to be involved in JNK signaling [7]. MAP3K1 (which is also known as MAPK/ERK kinase kinase 1,MEKK1) was one of the first MAP3K identified that is involved in the JNK activation [8]. MAP3Ks further activate the middle tier, which constitutes the Mitogen activated protein kinase kinases (MAP2Ks) via dual phosphorylation in the activation loop (T-loop) [1]. MKK4/MAP2K4 and MKK7/MAP2K7 are the MAP2Ks involved in the JNK signaling. They can activate JNKs by dual phosphorylation of Thr and Tyr residues [1], and these JNKs form the last tier of the pathway. JNKs further activate pro-apoptotic genes and apoptotic signaling through transactivation of transcription factors, such as c-Jun $[1,6]$. They can also phosphorylate and activate pro-apoptotic or anti-apoptotic proteins of the mitochondria [7].

Oxidative stress is a phenomenon that takes place when the pro-oxidant/anti-oxidant homeostasis of the cell is disturbed, resulting in severe oxidative damage to biomolecules and compromising the viability of the cell [9]. Sources of oxidative stress can be internal biochemical pathways (e.g., a large number of reactive oxygen species (ROS) are generated in the mitochondria as a part of the aerobic respiration process), as well as external factors, such as UV radiation, smoke, alcohol, and environmental pollutants [10]. Cells produce a wide range of anti-oxidant enzymes to neutralize these ROS species, and, in the physiological state, a balance exists between the production of ROS species and these enzymes, thus protecting the cells from oxidative damage $[10,11]$. However, when the concentration of pro-oxidants increases beyond the neutralization capacity of the anti-oxidants, it results in oxidative stress within the cells $[11,12]$.

Excess amounts of ROS species within the cell result in activation of Apoptosis signal-regulating kinase 1 (ASK1), a member of the MAP3K family, ultimately resulting in stimulation of JNK module and apoptosis (see Figure 1). The fate of the cell depends on the degree as well as duration of the activation signal, which, in this case, is oxidative stress [12]. In an unstimulated cell, ASK1 protein is bound to a redox sensitive regulatory partner, Thioredoxin (Txn), which prevents it from forming a homo-oligomer or a hetero-oligomer with ASK2 [12,13].

During oxidative stress, ROS species lead to oxidation of Cys-32 and Cys-35 residues within the redox center of Trx that results in the dissociation of this redox sensitive protein from ASK1, followed by the dissociation of 14-3-3ろ, thus allowing oligomerization of ASK1 [14]. Tumor necrosis factor receptor 
associated factors 2 and 6 (TRAF2 and TRAF6) are also activated and recruited to the ASK1 signalosome complex. ASK1, in its homo-oligomeric form, undergoes trans-autophosphorylation at the conserved Thr838 residue within the activation loop [14]. However, when it hetero-oligomerizes with ASK2, ASK2 undergoes auto-phosphorylation and further phosphorylates ASK1. Active ASK1 phosphorylates members of the MAP2K family, MKK4 and MKK7 [14]. These penultimate dual specificity kinases are responsible for activation of JNKs through phosphorylation of Thr and Tyr residues within the activation loop. Another set of proteins known as the JNK interacting proteins or JIPs (JIP1, JIP2, and JIP3) act as scaffold proteins, interacting with the three tiers of the JNK signaling pathway and thus are important for potentiating JNK activation [15].

Sustained JNK activation leads to the induction of apoptosis either through intrinsic or extrinsic pathway. In the intrinsic pathway, activated JNKs translocate to the mitochondria. Knockout studies in mouse embryonic fibroblasts (MEFs) suggested that JNK plays a critical role in the release of cytochrome $c$ from the inner mitochondrial membrane, which activates the Caspase-9 apoptotic cascade. However, the mechanisms by which JNK mediates the release of cytochrome $c$ are not completely understood [7]. The extrinsic pathway involves translocation of an active form of JNK to the nucleus, where it phosphorylates and transactivates many transcription factors. c-Jun was the first of JNK substrates to be identified and has been well characterized. JNK phosphorylates Ser63 and Ser73 residues within the N-terminal transactivation domain of c-Jun, thereby enhancing its transcriptional activity [2,16]. JNK also phosphorylates other components of the Activator protein 1 (AP1) complex, which includes Jun-B, Jun-D, c-Fos, and Cyclic AMP-dependent transcription factor ATF-2 (also known as Cyclic AMP-responsive element-binding protein 2, CREB2) [3,17]. Overall, more than 100 proteins have been reported as JNK substrates, which include 30 well-described TFs and transcriptional activator protein substrates. Therefore, JNK can regulate transcription of a large number of target genes through these transcription factors and generate a stress response in the cells [4].

Under normal physiological conditions, the JNK signaling cascade must remain inactive. Also, after the stress stimulus is removed, the cells need to return to their normal state. Hence, the JNK signaling pathway is tightly regulated. The components that are essential for the regulation of this pathway include the MAP3Ks, ASK1 and ASK2, the redox sensing Txn, JIPs, which are scaffold proteins, and dual specificity phosphatases [18]. Dual specificity phosphatases known as the Mitogen activated kinase phosphatases (MKPs) are important for down regulating JNK activity in cells post-stimulus.

The central dogma of structural biology states that, a protein needs to acquire its stable 3D folded state to perform its biological function. Many proteins need to have a stable structure to be functional, and these proteins are known as structured or globular proteins. Apart from these, the proteome of any organism also consists of biologically active, functional proteins that lack well-defined 3D structures either as whole or in some their parts. These proteins are referred to as intrinsically disordered proteins (IDPs) $[19,20]$ and hybrid proteins containing ordered domains and intrinsically disordered protein regions (IDPRs). Therefore, disorder can be global or local. Proteins which are completely disordered and have no structure at all are globally disordered, while proteins that have disordered as well as ordered regions are 
locally disordered [21]. Characteristic features of the amino acid sequence of intrinsically disordered proteins are biased composition of amino acids, low sequence complexity, abundance of hydrophilic and charged amino acids, and limited number of bulky and hydrophobic amino acids [20,22].

Many disordered proteins contain binding sites known as molecular recognition features (MoRFs).When they adhere to their specific partners, these proteins undergo a transition from disordered state to ordered state, in which the entire protein or a specific protein region adopts a well-structured conformation and is now ready to perform its function [22,23]. Disordered proteins are often involved in regulation, signaling and control processes. Their structural flexibility and plasticity enables them to interact with a multitude of binding partners, which are often unrelated to each other [24]. IDP/IDPRs contain large number of interaction motifs that allow them to bind to large number of targets. Therefore, many IDP/IDPRs function as hubs in numerous signaling pathways. Their flexible nature allows them to bind target molecules at multiple sites and form high-order complexes. Due to the presence of these multiple binding sites, IDP/IDPRs can play a role in allosteric responses, which are an intricate part of signaling processes $[19,21,22,24]$.

Proteins play a variety of crucial roles in maintenance of life, and hence their dysfunction can lead to occurrence of various pathologies [24]. IDPs and IDPRs, due to their unique structural and functional properties, are prone to misfold, which can lead to misidentification, misregulation, and missignalling and thus cause the development of diseases [24]. This is now known as the 'Disorder in disorders' or $D^{2}$ concept [24]. In line with this idea, many disease-associated proteins are known to be highly disordered. The misfolded peptides also tend to aggregate and gain toxic functions, as, for example is seen in various neurodegenerative diseases. Therefore, disordered proteins must be protected from pathological aggregation, since they comprise of few hydrophobic amino acids that can stick together and lead to pathogenesis.

In this study, we aim to identify the disordered regions in the proteins involved in the oxidative stress induced, extrinsic JNK signaling pathway using available computational tools and further characterize these disordered regions by identifying binding sites (MoRFs) and short linear motifs within these MoRFs, post-translational modification sites and protein-protein interaction networks.

\section{Materials And Methods}

\subsection{Retrieval of protein sequences}

Protein sequences of the various components of the JNK signaling cascade were collected from UniProt [25]. All the sequences used in the analysis are reviewed entries, and this assures that they have been manually curated and include functional annotations.

\subsection{Analysis of intrinsic disorder predisposition, disorder- based protein-protein interaction sites (MoRFs) and post-}




\section{translational modification sites}

In this study, we have used six disorder predictors, four from the PONDR® family (PONDR®VLXT, PONDR $®$ VSL2, PONDR®VL3 and PONDR®FIT) and two from IUPred (IUPred long and IUPred short) and a database of protein disorder $\left(D^{2} P^{2}\right)$. PONDRßVLXT [26], which uses a neural network-based approach to predict IDPRs in proteins is the first computational tool designed to predict disorder and is highly sensitive to local peculiarities within the protein sequence. PONDR®VSL2 [27] also uses neural networkbased algorithms to predict long and short disordered regions in protein sequences. PONDRßVL3 [28] accurately predicts long disordered regions in protein sequences. IUPRED [29] employs inter-residue total pairwise interaction energy to predict IDPRs using protein amino acid sequences. This algorithm has two implementations, IUPrd long and IUPred short to predict long and short disordered regions in protein sequences, respectively. PONDR $® F I T[30]$ is a meta predictor which combines the outputs of six different disorder predicts (PONDR®VSL2, PONDRßVLXT, PONDR®VL3, IUPred, TopIDP and Foldlndex) to reliably identify IDPRs. Using this combination of predictors makes PONDRßFIT even more accurate than its most accurate component.

The $\mathrm{D}^{2} \mathrm{P}^{2}$ database [31], holds predictions from six disorder predictors (PONDR®VLXT, PONDR®VSL2, IUPred, Espritz, PV2 and PrDOS) for more than 1765 proteomes and their variants. $D^{2} \mathrm{P}^{2}$ also shows predicted protein binding sites associated with disordered regions (MoRFs) along with various post translational modification (PTMs) sites and location of conserved functional domains (if any). In our study, we have used $D^{2} P^{2}$ to identify predicted MoRFs and PTMs within the disordered regions of query proteins.

\subsection{Identification of short linear motifs (SLiMs) associated with MoRF regions}

The Eukaryotic linear motif (ELM) database [32] has been used to identify conserved functional sequence motifs within the disordered regions of proteins known as SLiMs (short linear motifs). ELM subjects all identified SLiMs within the protein to undergo globular domain, structural, and context filtering to exclude SLiM matches that lie within the globular protein domains, which are highly structured, and this leaves behind SLiM from unstructured or disordered regions of the protein. The ELM server annotates these SLiMs into six categories, which include, motifs that serve as proteolytic cleavage sites (ELM_CLV), PTM sites (ELM_MOD), ligand binding motifs (ELM_LIG), motifs responsible for targeting to sub-cellular compartments (ELM_TRG), degron motifs for polyubiquitylation and protein targeting for proteosomal degradation (ELM_DEG) and docking motifs for modifying enzymes that are not within the active site (ELM_DOC). In this study, the SLiMs embedded into disordered regions that overlap with or are in the close proximity of the predicted MoRF residues (from $D^{2} \mathrm{P}^{2}$ ) have been identified and annotated using the aforementioned six categories.

\subsection{Identification of interacting partners of proteins involved in the JNK signalling cascade}


STRING (Search Tool for the Retrieval of Interacting Genes) [33] is a well-known database of known as well as predicted protein - protein interactions. It currently covers $24,584,628$ proteins from 5,090 different organisms. STRING has been used to identify interaction partners of proteins from the JNK signaling cascade as well the interaction of the proteins of this cascade with each other.

\subsection{Mapping of IDPRs and MoRFs on available PDB structures}

High resolution structures of proteins involved in the oxidative stress induced JNK signaling pathway were obtained from Protein data bank (PDB). The predicted IDPRs and MoRF regions were marked on the PDB structures of these proteins using UCSF Chimera [34].

\section{Results And Discussion}

\subsection{Global Overview of Intrinsic Disorder in proteins of the oxidative stress induced JNK signaling cascade}

In response to a wide range of environmental stimuli/stresses, such as oxidative stress, cells activate the JNK signaling pathway. The activated JNK MAPKs can influence a wide range of transcription factors (TFs), which affect expression of numerous specific genes within the cell. The degree and the duration of the environmental stress dictate the type of response and the fate of the cells. Figure 1 shows a schematic representation of the oxidative stress-induced JNK signaling cascade, whereas the actual roles of the 18 proteins involved in this pathway are described in Table 1. 
Table 1

Proteins involved in the extrinsic JNK signaling pathway induced by Oxidative Stress.

\begin{tabular}{|c|c|c|c|c|c|c|}
\hline $\begin{array}{l}\text { Sr. } \\
\text { No. }\end{array}$ & & Protein & $\begin{array}{l}\text { UniProt } \\
\text { ID }\end{array}$ & Length & $\begin{array}{l}\text { Role in JNK signaling } \\
\text { cascade }\end{array}$ & References \\
\hline 1 & MAP3Ks & ASK1 & Q99683 & 1374 & $\begin{array}{l}\text { Activates MAP2Ks (MKK4 } \\
\text { and MKK } 7 \text { ) by } \\
\text { phosphorylating residues Ser } \\
\text { and Thr in the conserved S-X- } \\
\text { A-K-T kinase motif. }\end{array}$ & [35] \\
\hline 2 & & ASK2 & 095382 & 1288 & $\begin{array}{l}\text { Forms a heterodimer with } \\
\text { ASK1 and activates ASK1 by } \\
\text { phosphorylation. }\end{array}$ & {$[36,37]$} \\
\hline 3 & $\begin{array}{l}\text { Regulatory } \\
\text { proteins of } \\
\text { MAP3Ks }\end{array}$ & Txn & P10599 & 105 & $\begin{array}{l}\text { Negatively regulates ASK1 } \\
\text { activation by inhibiting } \\
\text { formation of ASK1 } \\
\text { signalosome. }\end{array}$ & {$[38,39]$} \\
\hline 4 & & $\begin{array}{l}14-3- \\
3 \zeta\end{array}$ & P63104 & 245 & $\begin{array}{l}\text { Negatively regulates ASK1 } \\
\text { signalosome formation by } \\
\text { binding to phosphorylated } \\
\text { Ser967 and Ser964 of ASK1 } \\
\text { and ASK2 respectively. }\end{array}$ & [40] \\
\hline 5 & & TRAF2 & Q12933 & 501 & \multirow{2}{*}{$\begin{array}{l}\text { Stabilize ASK signalosome } \\
\text { complex and promote ASK1 } \\
\text { activation. }\end{array}$} & \multirow[t]{2}{*}[41,42]{} \\
\hline 6 & & TRAF6 & Q9Y4K3 & 522 & & \\
\hline 7 & \multirow[t]{2}{*}{ MAP2Ks } & MKK4 & P45985 & 399 & $\begin{array}{l}\text { Activator of p38 MAPKs and } \\
\text { JNKs. Activated MKK4 } \\
\text { phosphorylates Thr183 and } \\
\text { Tyr185 residues in JNKs. }\end{array}$ & [43] \\
\hline 8 & & MKK7 & 014733 & 419 & $\begin{array}{l}\text { Activates JNKs by } \\
\text { phosphorylating Thr183 and } \\
\text { Tyr185 residues in JNKs. }\end{array}$ & [44] \\
\hline 9 & \multirow[t]{3}{*}{ MAPK } & JNK1 & P45983 & 427 & \multirow{3}{*}{$\begin{array}{l}\text { Activate Transcription factors } \\
\text { like c-Jun, ATF2 and many } \\
\text { others, thus, influencing } \\
\text { transcriptional activity. }\end{array}$} & \multirow[t]{3}{*}[1,2]{} \\
\hline 10 & & JNK2 & P45984 & 424 & & \\
\hline 11 & & JNK3 & P53779 & 464 & & \\
\hline 12 & \multirow{3}{*}{$\begin{array}{l}\text { Scaffold } \\
\text { proteins }\end{array}$} & JIP1 & Q9UQF2 & 711 & \multirow{3}{*}{$\begin{array}{l}\text { Associates with MAP3Ks, } \\
\text { MAP2Ks, JNKs and MKPs } \\
\text { and regulate JNK activation. }\end{array}$} & \multirow[t]{3}{*}[5,45]{} \\
\hline 13 & & JIP2 & Q13387 & 824 & & \\
\hline 14 & & JIP3 & Q9UPT6 & 1336 & & \\
\hline 15 & \multirow{3}{*}{$\begin{array}{l}\text { Dual } \\
\text { specificity } \\
\text { phosphatases } \\
\text { (DSPs) }\end{array}$} & MKP1 & P28562 & 367 & \multirow{3}{*}{$\begin{array}{l}\text { Essential for determining } \\
\text { magnitude of JNK activation } \\
\text { and responsible for } \\
\text { downregulation of JNK } \\
\text { signaling cascade by } \\
\text { dephosphorylation of JNK }\end{array}$} & \multirow[t]{3}{*}[4,46]{} \\
\hline 16 & & MKP5 & Q9Y6W6 & 482 & & \\
\hline & & & & & & \\
\hline
\end{tabular}




\begin{tabular}{|lllllll|}
\hline $\begin{array}{l}\text { Sr. } \\
\text { No. }\end{array}$ & Protein & $\begin{array}{l}\text { UniProt } \\
\text { ID }\end{array}$ & Length & $\begin{array}{l}\text { Role in JNK signaling } \\
\text { cascade }\end{array}$ & References \\
\hline 17 & MKP7 & Q9BY84 & 665 & $\begin{array}{l}\text { after stress stimuli is } \\
\text { removed. }\end{array}$ & [47] \\
\hline 18 & $\begin{array}{l}\text { Transcription } \\
\text { factor }\end{array}$ & c-Jun & P05412 & 331 & $\begin{array}{l}\text { Activates AP-1 driven } \\
\text { promoters. }\end{array}$ \\
\hline
\end{tabular}

Table 2 and Figure 2 provide a global overview of the intrinsic disorder status of proteins involved in the oxidative stress-induced JNK signaling pathway. Table 2 contains the predicted percentage of intrinsic disorder (PPID) obtained for each protein from six disorder predictors, as well as their calculated $\mathrm{PPID}_{\text {Mean }}$ scores. Figure 2 is a $2 \mathrm{D}$ disorder plot representing the PPID $\mathrm{PONDR}^{\circledR}{ }_{\mathrm{FIT}}$ vs. PPID ${ }_{\text {Mean }}$ dependence for all the proteins analyzed in this study. Depending on the levels of intrinsic disorder, proteins can be classified into three groups, highly disordered ( PPID $\left._{\text {Mean }} \geq 30 \%\right)$, moderately disordered $\left(10 \% \leq \mathrm{PPID}_{\text {Mean }}\right.$ $<30 \%)$ and highly ordered (PPID Mean $^{<10 \%)}[48,49]$. 
Table 2

Predicted Percentage of Intrinsic Disorder (PPID) in proteins of the oxidative stress induced JNK signaling cascade.

\begin{tabular}{|c|c|c|c|c|c|c|c|c|}
\hline Sr. No. & Proteins & $\begin{array}{l}\text { PPID } \\
\text { VLXT }\end{array}$ & $\begin{array}{l}\text { PPID } \\
\text { VL3 }\end{array}$ & $\begin{array}{l}\text { PPID } \\
\text { VSL2 }\end{array}$ & $\begin{array}{l}\text { PPID } \\
\text { FIT }\end{array}$ & $\begin{array}{l}\text { PPID } \\
\text { IUPRED } \\
\text { long }\end{array}$ & $\begin{array}{l}\text { PPID } \\
\text { IUPRED } \\
\text { short }\end{array}$ & $\begin{array}{l}\text { PPID } \\
\text { Mean }\end{array}$ \\
\hline 1 & ASK1 & 28.09 & 31.44 & 33.62 & 17.54 & 10.63 & 9.75 & 21.32 \\
\hline 2 & ASK2 & 32.92 & 34.47 & 38.35 & 22.75 & 19.80 & 15.61 & 25.70 \\
\hline 3 & Txn & 11.43 & 38.10 & 30.48 & 20.95 & 0 & 10.48 & 14.29 \\
\hline 4 & $14-3-3 \zeta$ & 44.90 & 49.80 & 53.88 & 12.65 & 9.39 & 27.76 & 34.29 \\
\hline 5 & TRAF2 & 31.14 & 38.32 & 41.92 & 0 & 1.80 & 3.60 & 14.40 \\
\hline 6 & TRAF6 & 23.18 & 15.33 & 23.37 & 13.03 & 3.64 & 3.83 & 11.11 \\
\hline 7 & MKK4 & 27.57 & 26.32 & 37.59 & 22.81 & 22.56 & 23.31 & 27.57 \\
\hline 8 & MKK7 & 45.82 & 23.87 & 39.86 & 31.50 & 22.67 & 21.48 & 26.73 \\
\hline 9 & JNK1 & 19.67 & 23.65 & 27.63 & 15.46 & 16.86 & 17.80 & 19.44 \\
\hline 10 & JNK2 & 23.35 & 20.99 & 27.36 & 18.40 & 16.51 & 16.27 & 18.87 \\
\hline 11 & JNK3 & 14.87 & 19.61 & 26.72 & 15.52 & 15.52 & 14.87 & 14.87 \\
\hline 12 & JIP1 & 51.20 & 64.98 & 69.90 & 57.38 & 56.12 & 47.40 & 57.52 \\
\hline 13 & JIP2 & 58.01 & 73.67 & 73.79 & 68.20 & 65.05 & 56.31 & 65.66 \\
\hline 14 & JIP3 & 49.63 & 58.46 & 49.63 & 46.26 & 41.77 & 33.46 & 49.70 \\
\hline 15 & MKP1 & 20.16 & 14.17 & 23.98 & 13.90 & 0 & 3.81 & 11.72 \\
\hline 16 & MKP5 & 42.95 & 47.30 & 54.77 & 32.57 & 15.98 & 12.45 & 34.23 \\
\hline 17 & MKP7 & 37.44 & 41.95 & 55.49 & 39.10 & 26.47 & 27.97 & 36.84 \\
\hline 18 & c-Jun & 64.05 & 75.83 & 82.78 & 61.03 & 64.35 & 30.82 & 57.10 \\
\hline
\end{tabular}

From the PPID ${ }_{\text {Mean }}$ scores in Table 2, the 2D disorder plot in Figure 2 and the PPID based classification, we conclude that, none of the proteins involved in the JNK signaling cascade can be considered as highly ordered. Highest PPID ${ }_{\text {Mean }}$ of $65.7 \%$ was observed in case of the scaffold protein JIP2. The other scaffold proteins, JIP1 and JIP3 along with dual specificity phosphatases MKP5 and MKP7, and proteins 14-3-3 $\zeta$ and c-Jun were also found to be highly disordered. The MAP3Ks (ASK1 and ASK2), MAP2Ks (MKK4 and MKK7), MAPKs (JNK1, JNK2 and JNK3) as well as proteins Txn, TRAF2, TRAF6 and MKP1 belonged to the class of moderately disordered proteins. 
IDPRs and IDPs sometimes may contain short protein binding regions, which, upon coming in contact with appropriate binding partners, can undergo a transition from disordered to ordered state [23]. These regions are known as Molecular Recognition features (MoRFs) and can be predicted through algorithms, such as ANCHOR that recognize their distinct sequence signatures [50]. The $D^{2} P^{2}$ database identifies the MoRF regions in query proteins through ANCHOR. Using $D^{2} \mathrm{P}^{2}$, we have identified the number of MoRF residues and calculated their percentage (\% MoRF) for each protein (Table 3). The protein JIP2, which possesses the highest percentage of intrinsic disorder, also contains the highest percentage of MoRF residues. To gain further insight into the predicted IDPRs and MoRF regions, the ELM resource was used to predict short linear motifs (SLiMs) and their instances in the protein sequence. The ELM resource, after globular domain, structural and context filtering, retains the SLiMs which are associated with IDPRs. In Table 3, along with the MoRF residues and \% MoRF, the total (T) number of SLiMs identified and those retained $(\mathrm{R})$ after filtering have been listed. 
Table 3

Identification of SLiMs and MoRF regions in the proteins of the oxidative stress induced JNK signaling.

\begin{tabular}{|c|c|c|c|c|c|c|}
\hline \multirow{2}{*}{$\begin{array}{l}\text { Sr. } \\
\text { No. }\end{array}$} & \multirow[t]{2}{*}{ Protein } & \multicolumn{3}{|l|}{ ELM } & \multicolumn{2}{|l|}{ MoRFs } \\
\hline & & $\begin{array}{l}\text { Total (T) } \\
\text { /Retained } \\
\text { (R) }\end{array}$ & SLiMs & Instances & Residues (length) & $\begin{array}{l}\% \\
\text { MoRF }\end{array}$ \\
\hline \multirow[t]{2}{*}{1} & \multirow[t]{2}{*}{ ASK1 } & $\mathrm{T}$ & 95 & 625 & \multirow{2}{*}{$\begin{array}{l}8-36(29), 53-68(16), 1012-1020 \\
(9), 1213-1226(14)\end{array}$} & \multirow[t]{2}{*}{4.95} \\
\hline & & $\mathrm{R}$ & 80 & 401 & & \\
\hline \multirow[t]{2}{*}{2} & \multirow[t]{2}{*}{ ASK2 } & $\mathrm{T}$ & 94 & 557 & \multirow{2}{*}{$\begin{array}{l}17-27(11), 44-52(9), 833-839(7), \\
859-865(7), 876-890(15), 892-910 \\
(19), 937-951(15), 959-975(17), \\
988-1017(30), 1037-1046(10), 1076 \\
-1083(8), 1161-1170(10)\end{array}$} & \multirow[t]{2}{*}{10.71} \\
\hline & & $\mathrm{R}$ & 80 & 351 & & \\
\hline \multirow[t]{2}{*}{3} & \multirow[t]{2}{*}{ Txn } & $\mathrm{T}$ & 32 & 52 & \multirow[t]{2}{*}{ None } & \multirow[t]{2}{*}{0} \\
\hline & & $\mathrm{R}$ & 12 & 15 & & \\
\hline \multirow[t]{2}{*}{4} & \multirow{2}{*}{$\begin{array}{l}\text { 14- } \\
\text { 3_33 }\end{array}$} & $\mathrm{T}$ & 48 & 112 & \multirow[t]{2}{*}{$217-222(6)$} & \multirow[t]{2}{*}{2.45} \\
\hline & & $\mathrm{R}$ & 22 & 32 & & \\
\hline \multirow[t]{2}{*}{5} & \multirow[t]{2}{*}{ TRAF2 } & $\mathrm{T}$ & 59 & 157 & \multirow[t]{2}{*}{$244-251(8)$} & \multirow[t]{2}{*}{1.6} \\
\hline & & $\mathrm{R}$ & 44 & 91 & & \\
\hline \multirow[t]{2}{*}{6} & \multirow[t]{2}{*}{ TRAF6 } & $\mathrm{T}$ & 63 & 170 & \multirow[t]{2}{*}{ None } & \multirow[t]{2}{*}{0} \\
\hline & & $\mathrm{R}$ & 48 & 95 & & \\
\hline \multirow[t]{2}{*}{7} & \multirow[t]{2}{*}{ MKK4 } & $\mathrm{T}$ & 65 & 180 & \multirow[t]{2}{*}{$1-14(14), 29-62(34), 73-83(11)$} & \multirow[t]{2}{*}{14.79} \\
\hline & & $\mathrm{R}$ & 31 & 60 & & \\
\hline \multirow[t]{2}{*}{8} & MKK7 & $\mathrm{T}$ & 63 & 222 & 1 - 19 (19), 24 - 52 (29), 71 - 110 (40) & 21 \\
\hline & & $\mathrm{R}$ & 34 & 73 & & \\
\hline 9 & JNK1 & $\mathrm{T}$ & 72 & 215 & $319-324(6), 352-363(12), 377-387$ & 11.71 \\
\hline & & $\mathrm{R}$ & 54 & 102 & & \\
\hline 10 & JNK2 & $\mathrm{T}$ & 69 & 200 & 320 - 325 (6), 352 - $362(11), 391$ - 424 & 12.03 \\
\hline & & $\mathrm{R}$ & 48 & 100 & & \\
\hline 11 & JNK3 & $\mathrm{T}$ & 74 & 239 & $356-363(8), 390-399(10), 431-464$ & 11.21 \\
\hline & & $\mathrm{R}$ & 55 & 127 & & \\
\hline 12 & JIP1 & $\mathrm{T}$ & 72 & 323 & $\begin{array}{l}18-104(87), 118-202(85), 232-251 \\
(20) 259-323(65), 329-343(15)\end{array}$ & 46.69 \\
\hline & & $\mathrm{R}$ & 55 & 199 & $\begin{array}{l}369-403(35), 417-420(10), 458- \\
464(7), 477-484(8)\end{array}$ & \\
\hline
\end{tabular}




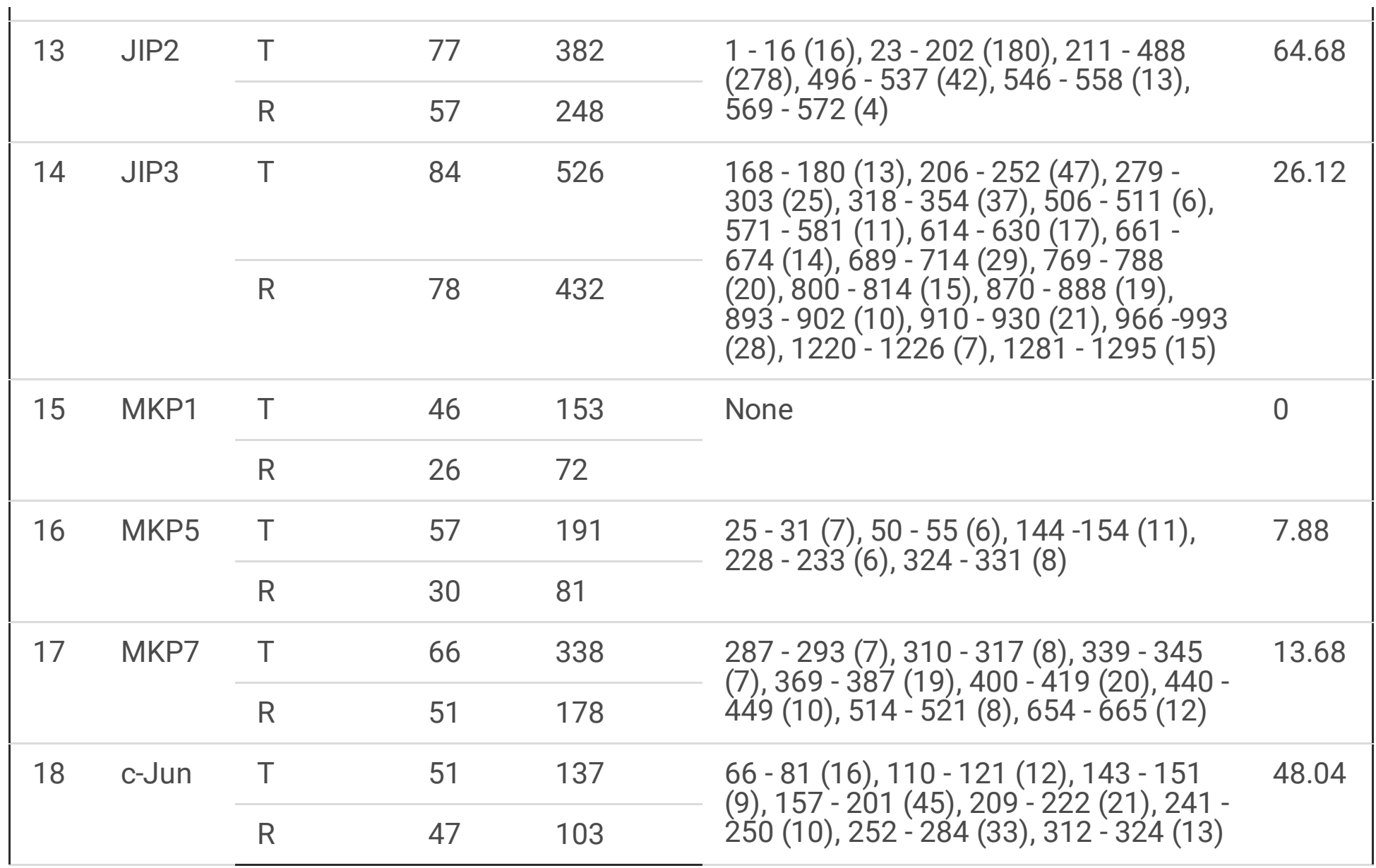

We have further analyzed the intractability of the 18 proteins involved in the JNK signaling cascade. The STRING database was used to obtain the inter-set and external interaction networks shown in Figure $3 \mathrm{~A}$ and 3B. The interaction network in Figure 3A clearly shows that all the 18 proteins are highly interconnected and engaged in a dense PPI network. The 18 nodes (proteins) in the network have 88 edges (connections), which is much higher than the expected edges ( $p$ value $<1.0$ e -16$)$ for a random set of proteins drawn from the human genome.

The average node degree, which indicates the number of connections each node has to the other nodes is 9.78. Next, we extended this PPI network further by including the second shell of 500 interactors to the first shell of 18 proteins. This extended PPI network is shown in Figure 3B. In order to generate a reliable network, the highest confidence score of 0.9 was set as the minimum required interaction score. The extended network included 518 nodes and 8125 edges. The number of expected edges calculated on a random protein set of comparable size was only 3002 ( $\mathrm{p}$ value $\left.<1.0 \times 10^{-16}\right)$. The average node degree for this network was 31.4 and was calculated centred at the 18 proteins involved in the JNK signaling cascade.

\subsection{Analysis of Intrinsic disorder in MAP3Ks of JNK signaling pathway}

\subsubsection{ASK1 (UniProt ID: Q99683)}


ASK1 is a 1,374 amino-acid-long protein and is also known as Mitogen activated protein kinase kinase kinase - 5 (MAP3K5). The domain structure of this protein includes a coiled coil domain at the N-terminal (CCl, residues 290-317), another coiled coil domain at the C-terminal (CCIl, residues 1236-1293) and a catalytic protein kinase domain (residues 670-940). ASK1 activation can be triggered in response to stressors, such as oxidative stress, ER stress, lipopolysaccharides, Fas ligand, GPCR agonists, inflammatory cytokines, and $\mathrm{Ca}^{2+}$ ion influx [51-55]. In normal conditions, ASK1 remains bound to Txn through the N-terminal region and 14-3-3Z at the phosphorylated Ser-967 [4]. But under oxidative stress, Txn undergoes oxidation and forms a disulphide bridge between Cys-32 and Cys-35 [4]. This oxidised form of Txn dissociates from ASK1 followed by the dissociation of 14-3-3ろ. This allows ASK1 to undergo a confirmation change and form homodimers in a head-to-tail fashion, which are stabilized by the CCII $[38,56]$. This ASK1 then undergoes phosphorylation at Thr-838 either by autophosphorylation or by ASK2 and thus gets activated [4]. Activated ASK1 phosphorylates MAP2Ks,such as MKK4 and MKK7, which further activate the JNK MAPKs [57]. ASK1 also plays a role in regulation of the JNK signaling.

According to the available literature, ASK1 has been known to be associated with a large number of human pathologies, which include neurodegenerative diseases, cancer and cardiovascular diseases, and hence has been a target for pharmaceutical intervention. Activation of ASK1 due to ROS under oxidative stress conditions has been known as the key mechanism for neurotoxicity induced by Amyloid $\beta$ peptide $(A B)$ in Alzheimer's disease (AD) $[58,59]$. Recent studies also showed that, knocking out ASK1 in mice injected with a-synuclein preformed fibrils lead to the reduction in the amount of phosphorylated asynuclein in striatum and cortex and attenuated neuroinflammatory reaction compared to the wild type animals. Hence, ASK1 inhibition could be used as a therapeutic strategy against Parkinson's disease (PD) [60].

A 2.10A resolution structure (PDB ID: 5ULM, Figure 4A1) of the central regulatory region (CRR, residues 269-658) of ASK1 (missing residues 269 - 271, 341, 383- 384,527 - 531, and 656 - 658) [61] and a $2.11 \AA$ resolution structure of the residues $660-977$ (PDB ID: 4BF2, Figure 4A2) of ASK1 [14], which includes the catalytic domain are available (missing residues $660-670,714-723,831-836$, and 941 977). Therefore, structure is currently available for $\sim 46 \%$ of the protein.

Figure 4A represents the disorder profile generated by six different disorder predictors and the PPID ${ }_{\text {Mean }}$ score for ASK1 is $21.3 \%$ (Table 2), which indicates that the protein is moderately disordered. Figure 4 shows that this protein contains 5 long IDPRs and 8 short IDPRs distributed across the entire sequence. The CCI domain (PPID Mean: 0\%) and the catalytic domain of ASK1 (PPID Mean: $0 \%$ ) are completely ordered, while the CCII domain is predicted to be highly disordered (PPID $\left.{ }_{\text {Mean }}: 66.1 \%\right)$. Small angle X-ray scattering (SAXS) data for the kinase/catalytic domain of ASK1 shows that the residues $659-669$ and $941-973$ are flexible and they overlap with our predicted IDPRs (residues $657-665,944-991$ ) [62]. D² $P^{2}$ also indicates the abundance of disordered regions in ASK1 (Figure 4A3).

Furthermore, $\mathrm{D}^{2} \mathrm{P}^{2}$ also shows that ASK1 has 4 short MoRF regions, which constitute $4.95 \%$ of the protein (Table 3). All MoRFs are embedded within IDPRs. ASK1 has 24 phosphorylation sites, out of which 10 lie 
in the disordered region, one acetylation site in the disordered region, 3 sumoylation sites and one nitrosylation site (Figure 4A3). ELM resource has predicted the presence of 95 SLiMs and their 625 instances, out of which $80 \mathrm{SLiMs}$ and their 401 instances are associated with disorder and hence were retained after filtering (Table 3).The SLiMs, which were found related to the predicted MoRF regions, have been identified and classified into six classes based on their function (Supplementary Table 2).

\subsubsection{ASK2 (UniProt ID: 095382)}

ASK2 is a 1,288-residue-long MAP3K belonging to the family of STE serine/threonine kinases. The gene coding for ASK2 generates 3 isoforms that are produced by the alternative splicing. ASK2 has $45 \%$ sequence identity to ASK1, and the domain structure of ASK2 is similar to that of ASK1 [49]. ASK2 consists of a protein kinase domain (residues 648-906) and two coiled coil (CC) domains, CCI (residues 1004-1029) and CCII (residues 1166-1205). It mainly differs from ASK1 at the $\mathrm{N}$ - and C-termini, as it is shorter at either ends than ASK1 [63]. No PDB structures for ASK2 have been reported as of yet.

ASK2 forms a heteromeric complex with ASK1, and this complex formation is essential for the effective expression and kinase activity of ASK2. It was observed that in the ASK1 deficient cells, endogeneous levels of ASK2 decreased as ASK2 underwent constitutive degradation [36,37]. Therefore, ASK1 is required for stability of ASK2 in cellular milieu. ASK2 in this complex was found to activate ASK1 by phosphorylation, therefore suggesting that ASK1 and ASK2 in the heteromeric complex facilitate each other's activities by distinct mechanisms [36,37]. Knockout studies have revealed that ASK2 is essential for oxidative stress-induced JNK signaling. ASK2, when in complex with ASK1, can function as a MAP3K and is competent to respond to oxidative stress, resulting in activation of JNK and p38 MAPK pathways $[36,37]$. ASK2 expression is reduced in cancer cells and tissues. In a recent study, it was observed that ASK2 in complex with ASK1 can work as a tumour suppressor by inducing pro-apoptotic activity in the epithelial cells [64].

The disordered regions in ASK2 predicted by six different disorder predictors and their mean have been indicated in the Figure $4 \mathrm{~B}$ and the PPID ${ }_{\text {Mean }}$ score of ASK2 was calculated to be $25.7 \%$ (Table 2). This indicates that ASK2 belongs to the category of moderately disordered proteins. As seen in ASK1, 3 long and 9 short disordered regions were observed to be scattered across the protein sequence in ASK2 as well. The CCI domain of ASK2 is completely disordered (PPID mean: $100 \%)$, CCIl domain is moderately disordered (PPID mean $: 30 \%)$ and the protein kinase domain (PPID mean $: 6.95 \%)$ is highly ordered. The result from $D^{2} P^{2}$ (Figure 4B1) also shows the prevalence of disorder in the protein.

According to $D^{2} P^{2}$, ASK2 has 12 MoRF regions which are distributed across the protein sequence and which constitute $12.3 \%$ of the protein and six of these regions are either embedded in or overlap with the predicted IDPRs (Table 3). The MoRF regions have been marked in the plot generated by $D^{2} P^{2}$ in Figure 4B1. $D^{2} P^{2}$ also shows that ASK2 has 11 phosphorylation sites, out of which 7 lie in the predicted IDPRs and 4 ubiquitylation sites, out of which two lie in the IDPRs. ASK2 has been predicted to have 94 SLiMs and their 557 instances, out of which 80 SLiMs and their 351 instances are disorder associated and were 
retained after filtering (Table 3). They have been further sorted with respect to their association with predicted binding sites/ MoRFs and have been described in Supplementary Table 2.

\subsection{Analysis of intrinsic disorder in proteins involved in regulation of MAP3Ks}

A large number ofASK1 accessory proteins have been reported. Several of these binding partners play important roles in regulation of ASK1 activity. For example, under normal conditions, Thioredoxin (Txn) and 14-3-3Z bind and negatively regulate ASK1 and thus prevent activation of JNK signaling cascade. However, under the oxidative stress, Txn and 14-3-3 3 dissociate and activate formation of the ASK1 signalosome by allowing dimerization of ASK1 and recruitment of TRAF2 and TRAF6 [39]. The detailed role of these proteins in the regulation of ASK1 along with analysis of their intrinsic disorder propensity and characterization of disordered regions are described in sections below.

\subsubsection{Thioredoxin (UniProt ID: P10599)}

Thioredoxin is a small redox protein, which is only 105-amino-acid-long. In fact, it is shortest protein analysed in this study. It exists as a homo-dimeric flavoprotein and is extremely important for regulation of redox homeostasis, growth, and differentiation within the cells [65]. Mammalian cells contain two thioredoxins, which are expressed ubiquitously. Trx-1 or Txn is mainly targeted to the cytosol and nucleus, whereas Trx-2 is present in the mitochondria [65,66]. Overexpression studies indicate that Trx-2 is important for regulation of mitochondrial ASK1. Activated ASK1 can induce cytochrome $c$ release and result in apoptosis without inducing JNK activation [67]. On the other hand, Trx-1/Txn is responsible for regulating the JNK signaling cascade through its interaction with ASK1.

The gene coding for human thioredoxin-1 (TXN) is located on chromosome 9q31. Coding region of $T X N$ gene spans $13 \mathrm{~kb}$ and is organized into 5 exons [68]. The active site of Txn is composed of Trp-Cys-GlyPro-Cys-Lys residues and is conserved across species. The two cysteine residues (Cys32 and Cys35) within the active site are important for ASK1 activation [69]. In normal conditions, Txn remains in a reduced state, and the cysteines form thiols. This reduced state of Txn strongly supports its binding to the ASK1 N-terminal coiled coil (NCC) region. Under oxidative stress, the cysteines within the active site form a disulphide, and in this oxidised state, Trx-1 has poor binding affinity for ASK1, which results in its dissociation from ASK1 [38,39]. This step is extremely crucial for the activation of ASK1.

Apart from its role in JNK signaling, Txn have also been implicated in a wide range of functions. One of the earliest ascribed functions of Txn includes its ability to act as a source of reducing equivalents for the enzyme ribonucleotide reductase [69] in prokaryotic systems. In eukaryotes, it is known to act as growth factor, antioxidant, regulator of transcription factors, and an inhibitor of apoptosis, but the mechanisms involved yet remain to be completely understood [70]. Txn has also been implicated in a wide range of diseases, such as atherosclerosis, AD, acquired immunodeficiency syndrome (AIDS), and cancer. In fact, elevated levels of Txn have been observed in a large number of cancers and are associated with 
aggressive growth of tumours [70]. Hence, Txn serves as a suitable target for drug development especially against cancers.

The structure of Txn homodimer has been determined through X-ray crystallography. A 0.94A resolution structure of mutated Thioredoxin homodimer (PDB ID: 4004) is available.

In Figure 5A1, the three mutated amino acids (residues $63,69,73$ ) in each monomer have been shown in cyan colour. Thus, $\sim 97 \%$ of the structure of Txn is known. Prediction of disordered regions in the protein has been carried out by six disorder predictors, and the results have been combined to compute PPID ${ }_{\text {Mean }}$ score (Table 2) for the protein which revealed that Txn has $15.2 \%$ disorder. Therefore, Txn belongs to the category of moderately disordered proteins. The per-residue disorder predisposition predicted by all six predictors and their mean has been indicated in Figure 5A. As per our analysis, the residues that comprise the active site of Txn are completely ordered and only short IDPRs have been observed in the N-terminal and $\mathrm{C}$-terminal regions of this protein. These findings are consistent with the crystallography studies that reveal the presence of a flexible arm in the C-terminal region of thioredoxin [65].

Results from the $D^{2} P^{2}$ platform do not show any MoRF residues in the protein (Table 3, Figure 5A2), but it indicates that Txn has six phosphorylation sites, out of which one lies in the disordered region, four acetylation sites out of which one is disordered, four ubiquitylation sites and three nitrosylation sites. Presence of $32 \mathrm{SLiMs}$ and their 52 instances in the protein has been predicted by ELM resource and after globular domain filtering, structural filtering and context filtering only disorder associated 12 ELMs and their 15 instances were retained.

\subsubsection{4-3-3ろ (UniProtID: P63104)}

The 14-3-3 family is a group of phospho-binding proteins and consists of seven isoforms $(\beta, Y, \varepsilon, \eta, \sigma, \tau$ and $\zeta)$, each of which are expressed by a different gene [71,72]. They may exist as homo or heterodimers [71] and are conserved across species. The unusual name of this protein is derived from its DEAEcellulose chromatography fraction number (14th fraction) and migration position on starch gel electrophoresis (fraction 3.3). It was initially described in 1967 by Moore and Perez as a brain abundant protein. But decades of research that followed have revealed the roles of the 14-3-3 family of proteins in regulation of every major cellular function, as they act as signaling hubs that integrate and control multiple signaling pathways, including cell cycle control, cell proliferation, apoptosis inhibition, glucose metabolism, autophagy and cell mobility [72].

In the JNK signaling pathway, 14-3-3Z acts as a negative regulator of ASK1 activation along with Txn. 143-3Z interacts with the phosphorylated Ser-967 and Ser-964 residues of ASK1 and ASK2 respectively. 143-3 3 first binds to the phosphorylated Ser-964 in ASK2, and ASK2 further recruits 14-3-3 3 to Ser-967 of ASK1, where it inhibits the formation of ASK1 signalosome [40]. The mechanism of this inhibition has not been completely understood, but according to the available evidence, it is likely that 14-3-3 3 either changes ASK1 conformation or blocks the active site. Under oxidative stress conditions, when the ROS 
levels are significantly increased, they trigger dephosphorylation of the serine residues and the subsequent release of 14-3-3Z allows ASK1 to undergo activation [73].

Localization studies on the 14-3-3 proteins indicate their presence in disease-associated inclusion bodies, such as neurofibrillary tangles in $A D$, Lewy bodies in PD, Lewy body-like hyaline inclusions in amyotrophic lateral sclerosis (ALS), pick bodies in Pick's disease, nuclear inclusions in spinocerebellar ataxia-1, and prion plaques in Cruetzfeldt-Jakob disease [74]. Although data from the invitro studies, genetic analysis, animal model studies, and post-mortem studies have made 14-3-3 proteins interesting therapeutic targets, their roles in neurological disorders need further investigation [75]. 14-3-3 3 is also known to be overexpressed in breast cancers. The role of 14-3-3 proteins as signaling hubs in many pro- survival signaling pathways suggests their immense potential as therapeutic targets for disruption of tumorigenic pathways [72].

Forty structures of 14-3-3ろ determined by X-ray crystallography are available on PDB. These multiple crystallography studies have failed to capture the flexible C-terminal region of 15 amino acids [72], which has also been predicted as disordered according to our analysis. A $2.0 \AA$ resolution structure (PDB ID: 1QJB) of the full-length 14-3-3 3 homodimer (missing residues 233 - 245) is shown in Figure 5B1 [76]. Thus, structure is known for $94.7 \%$ of this protein. Figure 5B represent disorder profile generated for $14-3-$ $3 \zeta$ by the six disorder predictors and their mean. The PPID ${ }_{\text {Mean }}$ score calculated for the protein is $34.29 \%$ (Table 2) which indicates that it belongs to the class of highly disordered proteins. Most of the disorder was observed at the N-terminal region of the protein, as two IDPRs of 40 and 26 amino acids respectively were identified (residues $1-40$ and 61 - 86) and another IDPR of 18 amino acids (residues 228 - 245) was predicted within the flexible $\mathrm{C}$-terminal region.

The result from $D^{2} P^{2}$ (Figure 5B2) shows that the protein has a single MoRF region of six amino acids, which constitutes $2.45 \%$ of the protein (Table 3 ). This predicted MoRF region is in close proximity to the C-terminal IDPR. D2P2 also indicates that the protein has 25 phosphorylation sites, out of which 6 lie in the disordered region, 11 acetylation sites, out of which 5 are disordered, 13 ubiquitylation sites, out of which 4 are disordered, 3 nitrosylation sites, out of which one lies in IDPR, and one methylation site. The result from the ELM resource predicts 48 SLiMs and their 112 instances in 14-3-3 $\zeta$ and after globular domain filtering, structural filtering and context filtering, 22 ELMs and their 32 instances are retained. The SLiMs associated with the predicted MoRF region have been described and further classified into six classes depending on their function (Supplementary Table 2).

\subsection{Tumour necrosis factor receptor (TNF-R) associated factors (TRAFs)}

The family of TRAF proteins contains seven members (TRAF1 - TRAF7), which are key signaling molecules involved in the regulation of a wide array of physiological processes. They were initially identified as adaptors for signaling that bind to the cytoplasmic region of receptors of TNF-R superfamily [77]. Further research led to the identification of more receptor families that use TRAFs. These include Toll 
like receptor (TLR), nucleotide binding oligomerization domain (NOD) - like receptor (NLR), RIG-I like receptor (RLR),T cell receptor, IL-1 and IL-17 receptors, IFN receptors and TGF $\beta$ receptors [77]. Studies with TRAF-deficient mice have helped to understand that TRAFs play physiological role in regulating embryonic development, bone metabolism, tissue homeostasis, immune system and stress response [77].

The members of the TRAF family (except TRAF1) possess a homologous domain at the N-terminal called the RING domain, zinc finger motifs, and a coiled coil domain. These proteins also possess a protein protein interaction domain (except TRAF7) or the TRAF domain at the C-terminal. This TRAF domain is divided into two subdomains, TRAF - $\mathrm{N}$ and TRAF - $\mathrm{C}$, which are composed of a coiled coil domain and seven to eight anti-parallel $\beta$-strand folds respectively $[78,79]$.

In the oxidative stress induced JNK signaling pathway, TRAF2 and TRAF6 are important for the formation of the ASK1 signalosome. Under the influence of ROS species, when Txn and 14-3-3Z are dissociated from ASK1, these two members of the TRAF family are recruited to form a high molecular mass complex [41]. The TRAFs stabilize the complex and promote the activation of ASK1. Although the precise mechanism by which TRAFs influence ASK1 activation is still unknown, studies have suggested that TRAF2 and TRAF6 result in homo-oligomerization of the N-terminal region and induce an open conformation in the central regulatory region of ASK1 $[41,42]$.

\subsubsection{TRAF2 (UniProtID: Q12933)}

TRAF2 is a 501-amino-acid-long protein coded by its gene located on chromosome 9q34.3. The fulllength structure of TRAF2 has not been determined yet. But a $1.90 \AA$ crystal structure of the RING domain (PDB ID: 3KNV) and the first zinc finger motif (Figure 6A1; residues 1 - 133; Missing residues 1 - 14) [80],

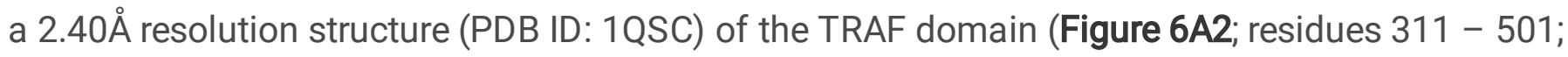
Missing residues 311 - 322) [81] and a 2.61 Åresolution structure (PDB ID: 3MOA) of the residues 267-328 are available (Figure 6A3) [82]. Therefore, structural information is currently available for almost $72 \%$ of the protein.

The per-residue disorder propensity predicted for TRAF2 by six disorder predictors and their mean are shown in Figure 6A. The disorder scores from each disorder predictor for the protein (Table 2) were averaged to obtain the PPID ${ }_{\text {Mean }}$ score of $14.4 \%$, indicating that TRAF2 belongs to the category of moderately disordered proteins. Three short IDPRs were observed in TRAF2, which included a 10-aminoacid-long region (residues 1 - 10) in the N-terminal segment, a short region (residues 314 - 318) within the coiled coil domain, and another short IDPR(residues 145, 147 - 155) between the RING domain and the coiled coil domain of the protein. As per our analysis, the RING domain (residues $34-72$ ) and the TRAF/MATH domain (residues 351 - 496) of the protein are completely ordered, while $10 \%$ disorder was observed in the coiled coil domain (residues 299 - 348). In a crystallography study, no electron density was observed for a short flexible region of 12 residues (residues 311 - 322) [81], and according to our analysis, the residues $314-318$, which lie within this flexible region, were also predicted to be disordered. 
The $D^{2} P^{2}$ database shows that TRAF2 possesses a single MoRF composed of 8 amino acids, which correspond only to $1.6 \%$ of the protein (Table 3 ). According to $D^{2} P^{2}$, the protein also possesses 12 phosphorylation sites, out of which two lie in the predicted IDPRs and 8 ubiquitylation sites (Figure 6A4). The ELM resource after globular domain filtering, structural filtering and context filtering, recognizes several SLiMs within the disordered region of a protein. In TRAF2, 51 SLiMs and their 157 instances were predicted. Out of these, 44 SLiMs and their 91 instances are retained after filtering (Table 3). The SLiMs, which are associated with the MoRF region, have been described and further classified into six classes according to their functions (Supplementary Table 2).

\subsubsection{TRAF6 (UniProtID: Q9Y4K3)}

Although complete structure of the TRAF6 protein has not been determined, $60 \%$ of its structure is known. A 2.20A $\mathrm{A}$ resolution structure (PDB ID: 3HCS) of the N-terminal domain of TRAF6 which includes the RING domain and the first zinc finger motif (residues 54 - 210) bound to the Ubiquitin conjugating enzyme (E2) is available [83]. We have evaluated the disorder propensity of the E2 protein in one of our previous studies [84]. A 1.80AD resolution structure (PDB ID: 1LB6) of the TRAF domain (residues 347 501) in complex with the protein CD 40 has been determined [85]. These above-mentioned structures have been represented in Figure 6B1 and figure6B2.

Combining the result obtained from six disorder predictors revealed that TRAF6 is a moderately disordered protein as it contains four short IDPRs which result in a PPID Mean $_{\text {score }}$ of $11.11 \%$ (Table 2). The Figure 6B shows the disorder score predicted for each residue of the protein by the six disorder predictors and their mean. According to our analysis, the RING domain (residues 70 - 108) and the coiled coil domain (residues 288 - 347) of TRAF6 are completely ordered, while the TRAF domain (residues 355 482 ) is predicted to have $2.34 \%$ of disordered residues. The extent of disorder in TRAF6 has also been depicted in the result from $D^{2} P^{2}$ (Figure 6B3). The database $D^{2} P^{2}$ indicates that the protein contains five phosphorylation sites and two ubiquitylation sites.No MoRF regions were predicted within the protein (Table 3). According to the ELM resource the protein has $63 \mathrm{SLiMs}$ and their 170 instances out of which $48 \mathrm{SLiMs}$ and their 95 instances are retained after globular domain, structural and context filtering (Table 3). Filtering helps to identify motifs that are associated with disordered regions.

\subsection{Analysis of Intrinsic Disorder in MAP2Ks of the Oxidative stress induced JNK signaling pathway}

MAP2Ks make up the second tier of the MAPK signaling pathways. They are Ser/Thr protein kinases belonging to the MAP kinase kinase subfamily. MAP2Ks are phosphorylated and activated by MAP3Ks, and they further activate the downstream MAPKs [1]. So far, seven MAP2Ks have been identified, and each of them possesses selective substrate specificity [86]. For example, although MKK7 is a specific activator of JNKs, MKK4 can activate both JNKs, as well as p38 MAPKs [87]. Studies showed that in the MKK7 deficient mice, JNK activation is completely lost even in presence of stress stimuli, but in the MKK4 
deficient mice, the activation of JNK is reduced to $50 \%$. Therefore, although MKK4 is responsible for the optimal activation of JNK, MKK7 is crucial for the activation of this protein [43].

Structurally, MKK4 and MKK7 are made up of the D (docking) domain, which as the name suggests, contains the docking site for substrate JNKs, the protein kinase domain, and the DVD (domain for versatile docking) domain, which harbours the conserved site for MAP3K binding. MKK7 also contains a single N-terminal coiled coil region [88]. MKK4 and MKK7 have a conserved Ser-Xaa-Ala-Lys-Thr (S$\mathrm{X}-\mathrm{A}-\mathrm{K}-\mathrm{T}$ ) kinase motif within their kinase domain. The Ser and Thr residues within this motif are the phosphorylation targets for MAP3Ks [35]. Activated MKK7 and MKK4 phosphorylate Thr-183 and Tyr-185 residues,respectively, within the JNK activation loop [43,44].

\subsubsection{MKK4 (UniProtID: P45985)}

MKK4 is a 399-residue-long protein encoded by the 11 exons of its gene, which lies on chromosome 17p12. Crystal structure of MKK4 (residues 80 - 399; Missing residues 80 - 82, 254-283, and 311-317) with a resolution of $2.30 \AA$ (PDB ID: $3 A L N$ ) containing the kinase domain and the DVD domain of the enzyme has been obtained and is represented in Figure 7A1 [89], indicating that structural coverage of this proteins is equal to almost $68 \%$.

The intrinsic disorder profile generated for MKK4 by six disorder predictors and their mean are shown in Figure 7A. Averaging the results from these disorder predictors, it was found that MKK4 has a PPID Mean score of $27.6 \%$ (Table 2), which puts this protein to the category of moderately disordered proteins. Our analysis shows that, the D domain (residues37-57) of MKK-4, which contains the docking site for JNK substrates, falls within a large, disordered region at the N-terminal (residues 1 - 85) of the protein and hence this domain is completely disordered ( PPID $\left._{\text {Mean }}: 100 \%\right)$. The protein kinase domain (residues102367 ) is moderately disordered, as it contains three short IDPRs (residues 133 - 134, 138 - 141, and 324 334), which make up $6.4 \%$ of the protein's total disorder, while the DVD domain (residues364-387), which harbours the conserved MAP3K binding site, is completely ordered (PPID $\left.{ }_{\text {Mean }}: 0 \%\right)$. In an X-ray crystallography study of the non-phosphorylated MKK4 (npMKK4) kinase domain in complex with AMPPNP and a ternary complex of npMKK4, AMP-PNP and p38 peptides, no electron density was observed for residues $80-83$ and the C-terminal residues 391 - 399 in the monomeric npMKK4 (residues 80 - 399) [89]. These residues were also predicted to be disordered according to our analysis.

The $\mathrm{D}^{2} \mathrm{P}^{2}$ plot (Figure 7A2) includes three disorder-associated binding regions, which constitute $14.8 \%$ of the protein (Table 3). All these regions are located within the 85-amino-acid-long $\mathrm{N}$-terminal disordered region. $D^{2} P^{2}$ also predicts that MKK4 has 12 phosphorylation sites, out of which 9 lie in the IDPRs, 2 ubiquitylation sites, out of which 1 is disordered, one di-methylation site located in the IDPR, and one acetylation site. The ELM resource has predicted $65 \mathrm{SLiMs}$ and their 180 instances within MKK4, and after the globular domain, structural and context filtering, $31 \mathrm{SLiMs}$ and their 60 instances were retained (Table 3). The ELMs, which are closely associated with the MoRF regions, have been described and classified based on their functions in Supplementary Table 2. 


\subsubsection{MKK 7 (UniProt ID: 014733)}

MKK7 is a 419-amino-acid-long kinaseencoded by a gene located on the chromosome 19p13.2. Structure of residues 103 - 419 (Missing residues 267 - 278, 404 - 419) of MKK7 with resolution 2.10 AD (PDB ID: 5B2L) has been obtained by X-ray crystallography and is available on PDB (Figure 7B1) [90]. This structure contains the kinase domain and the DVD domain of the protein. Thus, $69 \%$ of the protein structure of MKK7 is known.

Figure 7B represents the intrinsic disorder profile of MKK7 generated by six disorder predictors and their mean. Combining the disorder from all six different predictors, we observed that MKK7 has a PPID ${ }_{\text {Mean }}$ score of $26.7 \%$ (Table 2). Thus, the protein belongs to the category of moderately disordered proteins. Most of this predicted disorder lies in a long N-terminal IDPR (residues 1 -94), which contains the coiled coil region (PPID Mean $_{100 \%}$ ) and the D domain (PPID ${ }_{\text {Mean }}: 100 \%$ ) of MKK7. The protein kinase domain contains a short IDPR of 13 amino acids (residues 148 - 160) and four additional disordered residues and hence is mainly ordered (PPID Mean $: 6.9 \%$ ), while the DVD domain of MKK7 is completely ordered $\left(\mathrm{PPID}_{\text {Mean }}\right.$ 0\%). In a recent study, nuclear magnetic resonance (NMR) residual dipolar couplings and data from small-angle $X$-ray scattering were used to build ensemble models that describe secondary structure and identify partially structured regions in IDPs. The ensemble model for MKK7 concurs with our analysis and shows that the $\mathrm{N}$-terminal region of the protein is highly unstructured [91].

The $D^{2} P^{2}$ plot (Figure 7B2) indicates the presence of three MoRF regions, which constitute $21 \%$ of the protein (Table 3). These MoRF regions are associated with the long N-terminal IDPR as the first two regions (residues 1 - 19, 24 - 52) are embedded in the IDPR, while the third MoRF region (residues 71 110 ) overlaps with it. $D^{2} P^{2}$ also shows that this protein has 16 phosphorylation sites, out of which 7 are present in the disordered region and 3 ubiquitylation sites. According to the ELM resource, MKK7 has 222 instances of 63 predicted SLiMs. After filtering, 34 SLiMs and 73 instances that are associated with the disordered regions of the protein were retained. We have identified, described, and classified the SLiMs that are either embedded in or overlap with or are in close proximity with the predicted MoRF regions into six classes based on their functions (Supplementary Table 2).

\subsection{Analysis of intrinsic disorder in MAPKs of the oxidative stress-induced JNK signaling pathway}

JNKs are a group of evolutionarily conserved serine/threonine kinases belonging to the MAPK family. JNKs are mainly activated in response to extracellular stress stimuli and hence they are also known as Stress activated protein kinases. Alternative splicing of three genes that code for JNKs result in ten isoforms, out of which JNK1, JNK2, and JNK3 are predominant [1]. JNK1 and JNK2 are ubiquitously expressed, while JNK3 is mainly specific to neuronal cells [92]. The JNK protein was first identified for its role to phosphorylate Ser-63 and Ser-73 residues of c-Jun [16]. Now, more than 100 substrates of JNKs have been identified, and at least 30 of those are transcription factors [4]. 
Two important regions essential for JNK to perform its function include the activation TxYmotif and the protein kinase domain, which is responsible for catalytic activity of the protein. The upstream MAP2Ks, MKK4 and MKK7 phosphorylate JNK at the tyrosine and threonine residues of the TxY motif, respectively, and,therebyproduce activated form of JNK. This activated JNK then translocates into the nucleus, where it phosphoylates transcription factors, which include c-Jun and ATF2 of the activator protein -1 (AP-1) complex [1,2]. These activated TFs can then bind to promoters and enhancers of their target genes and mediate transcriptional activity [1,2].

The point whether JNK is pro-survival or pro-death is still controversial. However, temporal regulation has been indicated as a strong determinant in cellular response. Transient JNK activation results in cell survival, while prolonged activation of JNK results in apoptosis [93]. JNK1 has been implicated in type 2 diabetes-associated obesity and insulin resistance [94], along with JIP1 [95], while deregulation of JNK2 has been implicated in development of diseases, such as type I diabetes [96]. Thus, in recent studies, inhibitor molecules targeting JNK1 and JNK2 have been proposed as a therapeutic approach against diabetes $[96,97]$. JNK3 has been implicated in neurodegenerative diseases, such as AD and PD, and is therefore regarded as an attractive therapeutic target against neurodegeneration [92].

\subsubsection{JNK1 (UniProtID: P45983)}

JNK1 is a 427-amino-acid-long protein and the gene coding for it (MAPK8) produces 4 splice variants, but their functions have not been determined yet. A $1.33 \AA$ resolution structure (PDB ID: 2XRW, Figure 8A1) for JNK1 (residues 2 - 364) was obtained using X-ray diffraction [98] (missing residues 173-188), indicating that the structure is known for $81.26 \%$ of JNK1. Averaging the disorder content obtained by six different predictors, we observed that JNK1 has a PPID ${ }_{\text {Mean }}$ score of $19.4 \%$ (Table 2). Thus, JNK1 belongs to the category of moderately disordered proteins. The protein kinase domain (residues $26-321$, PPID $_{\text {Mean }}$ : $0 \%$ ) and the TxY activation motif (residues $183-185$, PPID $_{\text {Mean }}$ : $0 \%$ ), which are extremely crucial for protein function are completely ordered. A long IDPR of 62 amino acids is present in the C-terminal region (residues 366 - 427) of the protein. JNK1 also contains two short IDPRs, one of which lies at the Nterminal and the other is in close proximity of the protein kinase domain. Figure8A represents the disorder profile containing the outputs of six per residue disorder predictors along with their mean.

The $D^{2} P^{2}$ plot shows the distribution of MoRF regions in JNK1 (Figure 8A2) and according to it, the protein has 5 MoRF regions, which constitute $11.7 \%$ of its length (Table 3 ). Three MoRF regions are embedded within the long IDPR at the C-terminal segment of JNK1. The protein contains 13 phosphorylation sites, out of which one lies in the IDPR, two ubiquitylation sites, one acetylation site, and one nitrosylation site (Figure 8A2). According to the ELM resource, the protein has 215 instances of 72 SLiMs,out of which 54 SLiMs and their 102 instances are retained after globular domain, structural and context filtering. The retained SLiMs instances, which are associated with the predicted MoRF regions of the protein have been classified and described further (Supplementary Table 2).

\subsubsection{JNK2 (UniProtID: P45984)}


JNK2 is a protein made up of 424 amino acids encoded by the MAPK9 gene, which gives rise to four splice variants. The functions of the splice variants have not been determined yet. Through $\mathrm{X}$ ray crystallography, a $2.14 \AA$ resolution structure (PDB ID: 3E70, Figure 8B1) of the residues 1-361 (Missing residues $1-8,282$ - 283, 338 - 346), which included the catalytic protein kinase domain (residues 26 321) and the TxY activation motif(residues 183 - 185) of JNK2 was determined [99]. Thus, the structural coverage of this proteins is equal to $80.7 \%$.

The per-residue disorder profiles generated for JNK2 by six different predictors and their mean are shown in Figure 8B. The PPID ${ }_{\text {Mean }}$ score for this protein was calculated to be $18.9 \%$.These values indicate that JNK2 belongs to the category of moderately disordered proteins. As observed in JNK1, the TxY motif in JNK2 is completely ordered, while the protein kinase domain, which was completely ordered in JNK1, contains $2.7 \%$ disorder in JNK2. The short IDPR at the N-terminal and the long IDPR at the C-terminal segments that were observed in JNK1 are also seen in JNK2 (residues 1 - 6and $367-424$, respectively). Besides these, the protein contains two short IDPRs out of which one is embedded in the kinase domain (residues 286 - 293).

According to the $\mathrm{D}^{2} \mathrm{P}^{2}$ database (Figure 8B2), JNK2 has three MoRF, which constitute $12 \%$ of the protein length (Table 3). One of these MoRF regions is embedded within the C-terminal IDPR. The $\mathrm{D}^{2} \mathrm{P}^{2}$ plot (Figure 8B2) also shows that the protein has ten phosphorylation sites, out of which three lie in IDPRs, one nitrosylation site, three ubiquitylation sites, and one acetylation site. The ELM resource predicts the presence of 200 instances of 69 SLiMsin JNK2, and 48 of these predicted SLiMs and their 100 instances are associated with disordered regions and hence, were retained after filtering. Based on their association with the predicted MoRF regions and their function, these SLiMs were further classified into six groups (Supplementary Table 2).

\subsubsection{JNK3 (UniProtID: P53779)}

These 464-amino-acid-long protein is encoded by the MAPK10 gene, which gives rise to two alternatively spliced isoforms. However, the functions of the splice variants are not known as of yet. A $2.10 \AA$ resolution structure of the full length JNK3 (PDB ID: 3TTJ; Missing residues $1-45,54-55,212-216$, $221-224,373-383,401$ - 464) was obtained by X-ray crystallography (Figure 8C1) [100]. Thus, structure is known for $71.8 \%$ of this protein.

Figure $8 \mathrm{C}$ shows the disorder propensity predicted for each residue of JNK3 by six disorder predictors and their mean. The PPID ${ }_{\text {Mean }}$ score for JNK3 was calculated to be $14.87 \%$ and Thus, JNK3, like JNK1 and JNK2, belongs to the category of moderately disordered proteins. Similar to JNK1 and JNK2, a long IDPR of 63 amino acids (residues 403 - 465) was observed at the C-terminal part of the protein. The protein also possesses a short IDPR of 6 amino acids (residues 375 - 380). As observed in JNK1, the protein kinase domain (residues 64 -359) and the TxY activation motif (residues $183-185$ ) are completely ordered in JNK3 as well. Using the ANCHOR algorithm, the $\mathrm{D}^{2} \mathrm{P}^{2}$ database has predicted three MoRF regions in the protein, which constitute $11.2 \%$ of its length (Table 3 ), and one of these regions (residues 
431 - 464) is embedded within the long IDPR at the C-terminal. The $D^{2} P^{2}$ plot also shows that the JNK3 has 8 phosphorylation sites, one ubiquitylation site, and one acetylation site (Figure $8 \mathrm{C} 2$ ). According to the ELM resource, the protein has 54 SLiMs and their 239 instances, out of which 55 SLiMs and 127 instances, which are disorder-associated being retained after filtering. The retained SLiMs which are embedded in, overlap with, or are in close proximity of the predicted MoRF regions were identified and classified based on their functions (Supplementary Table 2).

\subsection{Analysis of intrinsic disorder in scaffold proteins 3.7.1. JNK interacting protein 1 (JIP1; UniProtID: Q9UQF2)}

JIP1 is a 711 amino acid long protein belonging to the family of JNK interacting scaffold proteins. The JIP1 gene is located on chromosome 11 while a JIP1 pseudogene is located on chromosome 17 (17q21) [15]. Apart from a JNK binding domain (residues 127-285), the protein also contains a $\mathrm{SH}_{3}$ domain (residues 488-549) and a phosphotyrosine binding domain (PTB domain, residues 561 - 700) which are both located within the $\mathrm{COOH}$ terminal region. The structure of the full length JIP1 has not been determined as of yet. A $1.99 \AA$ (PDB ID: 4H39, Figure 9A1) structure of a short JIP1 peptide (residues 158167) in complex with JNK 3 [101] and $2.70 \AA$ A structure (PDB ID: 6FUZ, Figure 9A2) of the C-terminal peptide of JIP1 (residues 701 -711, Missing residues 701 - 703) in complex with Kinesin light chain-1 (KLC-1) [102] are available. Therefore, structure is known only for $2.5 \%$ of the JIP1 sequence.

JIP1 and JIP2 are mainly expressed in neurons and in insulin secreting $\beta$-cells of pancreas [1]. JIP1 is essential for the stress-mediated activation and modulation of JNK signaling. It associates with proteinkinases as well as protein-phosphatases that target JNK and regulates their activity, as well as subcellular localization $[5,45]$. Transfection assays in the cell culture studies and studies in mouse model [103] have helped determine the roles of JIP1 in JNK activation. Deletion of Jip1gene in mouse models leads to the reduced apoptosis of hippocampal neurons in response to stress. This is an indication of decreased JNK activity and reduced c-Jun phosphorylation [103]. JIP1 has also been reported to bind top190 Rho guanine nucleotide exchange factor (RhoGEF) [104], Apolipoprotein E receptor-2 (ApoER2) [105], LDL-receptor mediated protein, and megalin[106]through its PTB domain. JIP1 also interacts with Kinesin-1 [107] and p150 Glued, which is a subunit of the dyenein-dynactin complex [108]. By binding to these partners, JIP1 co-ordinates and regulates amyloid precursor protein (APP) transport by switching between anterograde and retrograde motile complexes in neurons and, thereby, determines the directionality of axonal transport of APP [108]. Impairment of theaxonal transport can lead to the excessive production and aggregation of $A \beta$ peptide into senile plaques, thus, resulting in $A D$ [108].

Multi-parametric disorder analysis of JIP1 was conducted in our study using six disorder predictors (see Figure 9A). Averaging the output of these predictors revealed that JIP1 has a PPID Mean score of $57.5 \%$ (Table 2). This clearly places JIP1 in the category of highly disordered proteins. Figure9Arepresents the per-residue disorder predisposition of JIP1 predicted by all six disorder predictors and their mean. According to our analysis, the protein contains a large 309-residue-long IDPR (residues 67 - 375), and the 
JNK binding domain is completely disordered as it is embedded within this region. JIP1 also contains another long IDPR of 41 amino acids (residues 414 - 454) and 4 short IDPRs (residues 1 - 24, 32 - 58, 60 $-64,709$ - 711).

The $D^{2} P^{2}$ plot also illustrates the abundance of disordered regions in JIP1 (Figure 9A3). It also shows that JIP1 has 9 MoRF regions predicted by the ANCHOR algorithm, which constitute $46.7 \%$ of the protein (Table 3). Among the predicted MoRF regions, four are found to be embedded into the 309-amino-acidlong IDPR and three overlaps with other predicted IDPRs. Also, according to $D^{2} P^{2}$, the protein has 40 phosphorylation sites, out of which 31 lie in the disordered regions, 1 methylation, and 1 acetylation site both located within the disordered regions. The ELM resource has predicted the presence of 323 instances of 72 SLiMsin JIP1, and among these 55 SLiMs and their 199 instances are linked to disorder as they are retained after globular domain, structural, and context filtering (Table 3). The retained SLiMs, which are associated with predicted MoRF regions, were further classified into six classes based on their function (Supplementary Table 2).

\subsubsection{JNK interacting protein 2 (JIP2; UniProtID: Q13387)}

JIP2, a protein belonging to the family of JNK interacting proteins, is 824-amino-acid-long and shows highsequence homology to JIP1[5]. Domain structure of JIP2 is similar to that of JIP1. This protein has a JNK-binding domain in the $\mathrm{N}$-terminal region (residues 110 - 275) and a $\mathrm{SH}_{3}$-domain (residues 604-665) and a PTB domain (residues 677 - 813) in the C-terminal region [15]. Three isoforms of this protein with length of 797, 598, and 443 amino acids have been identified. The gene coding for JIP2is located within the chromosome 12 (12q13) and is made up of 12 exons[15]. No PDB structure of this protein is available. Similar to JIP1, this protein is expressed in neurons and insulin-secreting $\beta$-cells of the pancreas [1] and in testis [5].

Unlike other JIP proteins that form homo-oligomers, JIP2 has been reported to form hetero-oligomers with JIP1 and JIP3. JIP2 interacts with multiple components of the JNK signaling pathway and plays a role in JNK activation [109]. It has also been reported to interact with isoforms of the MAPK p38. The interaction of JIP2 with proteins of the APP family is highly conserved, and JIP1 and JIP2 collectively play a role in regulating the phosphorylation of APP by JNK [110]. Studying these interactions is important to gain insight into the molecular mechanisms involved in AD.

Figure 9B represents the per-residue disorder predisposition of JIP2predicted by six IDP predictors and their mean. Combining the output from these predictors indicates that JIP2 belongs to the category of highly disordered proteins as itsPPID ${ }_{\text {Mean }}$ score was calculated to be $65.7 \%$ (Table 2).

Three long IDPRs made up of 155 amino acids (residues 1 - 155), 339 amino acids (residues 166 - 504), and 41 amino acids (residues 530 - 570) were predicted along with three very short IDPRs (residues 157 159,627 , and 823 - 824). According to our analysis, the JNK binding domain of JIP2 is highly disordered, while the $\mathrm{SH}_{3}$ domain and PTB domain are mostly ordered. 
As no entry for JIP2 was available in $D^{2} P^{2}$, we used the ANCHOR algorithm which is available at (https://iupred2a.elte.hu/) directly to predict MoRF regions, but the locations of the PTM sites in this protein have not been documented. ANCHOR predicts that JIP2 has 6 MoRF regions, which make up $64.7 \%$ of its sequence (Table 3 ). All these MoRFs are either embedded in or overlap with the predicted IDPRs. The ELM resource has predicted 382 instances of 77 SLiMs, out of which only 57 SLiMs and their 248 instances are retained as linked to disordered regions. Furthermore, the SLiMs associated with the predicted MoRF regions were identified and classified into six classes based on their function

(Supplemetary Table 2).

\subsubsection{JNK interacting protein 3 (JIP3; UniProt ID: Q9UPT6)}

JIP3 is another protein belonging to the family of JNK interacting protein and is 1,336-amino acid-long. Its sequence is distinct from those of JIP1 and JIP2 but is highly related to JIP4 [5,109]. JIP3 is mainly expressed in the brain and is also expressed in testis and heart and in other tissues at a low level [109]. It can form homo-oligomers and hetero-oligomers with JIP2. JIP3 binds to the MLK3-MKK7-JNK signaling module and activates JNK signaling [111]. A splice-variant of JIP3 also acts as a scaffold protein. JIP3 is localized mainly to the cytoplasm and accumulates in the growth cones of developing neuritis [111]. A recent study has shown that JIP3 enhances axonal elongation in hippocampal and cortical neurons in a kinesin and JNK dependant manner [112]. This elongation is facilitated by JIP3 mediated JNK activation at the tip of axons and is modulated by the dynamics of actin filaments and cofilin activity [112].

The domain structure of JIP3 includes RILP homology (RH1) domain (residues12-100), which overlaps with the Kinesin binding domain (residues50-80) and coiled coil I region (residues58-177), JNK binding domain (residues209-225), and Coiled coil II (residues440-554) region, which overlaps with both leucine zipper domain (residues423-458) and RH2 domain (residues520-594) [109,112]. A structure of the fulllengthJIP-3 is not available as of yet, but a $2.06 \AA$ resolution structure of the leucine zipper II domain of JIP3 (PDB ID: 4PXJ, Figure 9C1) has been determined [113].

Figure $9 \mathrm{C}$ shows that the JIP3 has several long IDPRs, which overlap with functional domains. The coiled coil I (PPID mean: 69.2\%) and coiled coil II (PPID mean: 80.9\%) regions, the kinesin binding domain (PPID mean: $41.9 \%)$, RH1 (PPID mean $_{3}: 37.1 \%$ ) and RH2 (PPID mean $: 69.3 \%$ ) domains are highly disordered and the leucine zipper domain (PPID mean $: 11.1 \%$ ) is moderately disordered, while the JNK binding domain (PPID mean $: 0 \%$ ) is highly ordered. The protein JIP3 has a PPID ${ }_{\text {Mean }}$ score of $49.70 \%$. Our prediction puts JIP3 in the category of highly disordered proteins. The result from $D^{2} P^{2}$ (Figure 9C2) also reveals the prevalence of disorder in JIP3. It shows the presence of 20 MoRF regions in JIP3, which make up $26.12 \%$ of the protein. Seven of these MoRFs are embedded within the predicted IDPRs, while four overlap with IDPRs (Table 2). D2 $P^{2}$ further indicates that JIP-3 has 16 phosphorylation sites out of which 9 lie in the disordered region, 3 methylation sites and 1 ubiquitylation site in the disordered region (Figure 9C2). According to ELM, JIP3 is predicted to have 526 instances of 84 SLiMs. There are 432 instances of the 78 disorder-linked SLiMs, which are retained after the globular domain, structural, and context filtering. The 
retained SLiMs associated with MoRF regions have been identified and classified in Supplementary Table 2.

\subsection{Analysis of intrinsic disorder in MAP kinase phosphatases (MKPs)}

The outcomes of MAPK signaling pathways in cells depend on the duration and magnitude of the stress signals that are responsible for the kinase activation. But when the stress is removed, downregulation of MAPKs is achieved through dephosphorylation by tyrosine phosphatases, serine/threonine phosphatases, and dual specificity phosphatases (DSPs) $[114,115]$. MKPs are a group of DSPs that possess the ability to dephosphorylate phophotyrosine, as well as phophoserine/phosphothreonine residues within MAPKs of JNK, ERK, and p38 signaling pathways [114-116].

So far, 10 catalytically active members of the MKP family have been identified in mammals (MKP1, MKP2, MKP3, MKP4, MKP5, MKP7, MKPX, PAC1, hVH3, and hVH5), and they have been classified based on their sub-cellular localization. MKP1, MKP2, hVH3, and PAC1 are present in the nucleus; MKP3, MKP4, and MKPX are present in the cytoplasm, while MKP5, MKP7, and hVH5 are found in both nucleus and cytoplasm [117]. All MKPs contain a highly conserved C-terminal DSP catalytic domain (DSPC). They also contain an N-terminal Rhodanese domain, which is thought to contain crucial Kinase interacting motifs (KIMs) that confer MAPK substrate specificity [115]. MKP1, MKP5, and MKP7 are known to show specificity for JNKs and p38 MAPKs over ERKs [115,117].

MKP1, MKP5, and MKP-7 coordinate with each other and regulate the magnitude and persistence of JNK activation in response to oxidative stress [118]. Overexpression of MKP1 in mouse embryonic fibroblast cells shows increased resistance of cells against oxidative damage. Therefore, MKP1 activation is a survival mechanism against oxidative damage [119]. But even MKP1-based JNK inactivation is dependent on the magnitude and persistence of the oxidative stress. A study using RNA interference for characterizing functional role of MKPs has shown that MKP1 and MKP5 are essential for determining the magnitude of oxidative stress induced JNK activation, whereas MKP7 shows significant late phase induction and is responsible for downregulation of the JNK signaling cascade by dephosphorylation of JNK [118].

\subsubsection{MKP1 (UniProt ID: P28562)}

MKP1, also known as DSP1, is a 367-amino-acid-long protein (39.30 kDa) encoded by the gene located on the chromosome 5q34. A $2.23 \AA$ resolution crystal structure (PDB ID: 6D66) is known for the catalytic domain (DSPC) of MKP1 fused with the Maltose binding periplasmic (MBP) protein and in complex with designed AR protein mbp 3_16 (Figure 10A1). The MBP protein is a fixed arm crystallization chaperone, which is often used to assist crystallization of other proteins [120]. Structure is known only for $39 \%$ of this protein. 
Figure 10A represents the per residue disorder propensity of MKP1 predicted by six disorder predictors

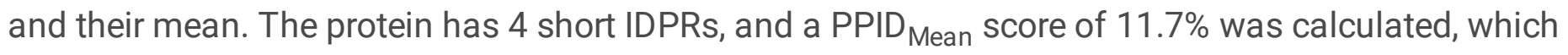
places the MKP1 to the category of moderately disordered proteins. Short IDPRs of 5 and 10 amino acids were observed at the $\mathrm{N}$ - and $\mathrm{C}$-termini of the protein respectively. The RhoD domain and the DSPc domain of the protein did not contain any disordered residues, and hence were completely ordered. A disordered region of 27 amino acids located between the RhoD domain and the DSPc domain, where it serves as a flexible linker. The $D^{2} P^{2}$ database (Figure 10A2) did not show presence of MoRFs in MKP1 (Table 3). But it indicates the presence of 7 phosphorylation sites, of which five are disordered, 3 ubiquitylation sites, and one acetylation site. The ELM resource has retained disorder-associated 72 instances of 26 SLiMs after filtering from the total of 153 instances of 48 SLiMs. As no MoRF regions were observed in this protein, no MoRF-associated ELMs were identified (Supplemtary Table 2).

\subsubsection{MKP5 (UniProtID: Q9Y6W6)}

MKP5 is also known as DSP10, and the gene located on chromosome 1q41 codes for this $52.64 \mathrm{kDa}$ protein. A $2.20 \AA$ structure (PDB ID: 2OUC) of the MAPK binding domain (residues $148-287$, Missing residues 162 - 171) and a $2.80 \AA$ resolution structure (PDB ID: 2OUD) of the catalytic domain of MKP5 (residues 315 - 482) have been determined using X-ray crystallography (Figure 10B1and10B2) [121]. Thus, structure is known for $61.8 \%$ of this protein.

The disorder profile for MKP5 shown in Figure 10B represents the per-residue disorder propensity of the protein predicted by six disorder predictors and their mean. As per this analysis, MKP5 has a PPID ${ }_{\text {Mean }}$ score of $34.23 \%$ (Table 2), which puts it in the category of highly disordered proteins. MKP5 contains 8 IDPRs, which are spread across the protein. The Rhodanese (RhoD) domain, which is also the MAPK binding domain, contains three short IDPRs, which constitute $14.4 \%$ of the total disorder of the protein. Another IDPR of 33 amino acids lies in close proximity of the RhoD domain. The catalytic domain (DSPC) of the protein was found to be completely ordered.

The expression construct used in crystallization experiments for the MAPK binding domain contained 9 additional residues (residues 139 - 147) which did not show any electron density and hence were regarded as disordered [121]. These residues were also predicted to be disordered according to our analysis.

According to the $D^{2} P^{2}$ database (Figure 10B3), MKP5 has 5 short MoRF regions, which constitute $7.9 \%$ of the protein, and three of these are associated with the predicted IDPRs (Table 3). Presence of three phosphorylation sites in MKP-5 was also predicted out of which two lie in the disordered regions. The ELM resource has predicted the presence of 191 instances of 57 SLiMsin the protein, and after globular domain, structural, and context filtering, only 81 instances of 30 disorder-associated SLiMs were retained. The SLiMs, which are either embedded in or overlap with or are in close proximity of the MoRFs were identified, and these MoRF-associated SLiMs have been described in Supplementary Table 2.

\subsubsection{MKP7 (UniProtlD: Q9BY84)}


Alternative nomenclature for the protein MKP7 is DSP16, and the gene encoding this $73.10 \mathrm{kDa}$ protein is located on chromosome 12p13.A crystal structure of the RhoD domain of MKP-7 (residues 5 - 150; Missing residues 139 - 150) with a resolution of $2.20 \AA$ (PDB ID: 2VSW) has been determined (Figure 10C1). Another structure of the MKP7 residues 156 - 301 (Missing residues 156 - 157, 301) in complex with JNK1 (PDB ID: 4YR8) is also available (Figure 10C2) [116]. Thus, structure is currently known for only $41.65 \%$ of this protein.

The per residue disorder propensity of MKP7 predicted by six disorder predictors is shown in Figure 10C along with their mean. The PPID ${ }_{\text {Mean }}$ score for this protein was calculated to be $36.8 \%$, which classifiesMKP7 as highly disordered proteins (Table 2). The N-terminal part of MKP7 is highly ordered, as it only contains a short IDPR of 5 amino acids. The RhoD domain, which is responsible for the MAPK substrate specificity, and the DSPc domain, which harbours the catalytic site of the protein, are highly structured as well, since none of the residues within these domains fall into IDPRs. It was observed that most of the second half of MKP7 is disordered, as it contains three large IDPRs of 98 (residues 302 399), 69 (residues 440 - 508), and 73 (residues 593 - 665) amino acids respectively.

The $D^{2} P^{2}$ plot (Figure 10C3) has indicated the presence of 8 short MoRF regions in MKP7, which make up $13.68 \%$ of the protein (Table 3 ). Interestingly, six of these regions are associated with the predicted IDPRs. The 98-amino-acid-long IDPR contains three MoRFs embedded in it and one MoRF region is in its close proximity. The 69- and 73-amino-acid-long IDPRs have one MoRF embedded in each of them. $\mathrm{D}^{2} \mathrm{P}^{2}$ also indicates that MKP7 has 8 phosphorylation sites, and six of them lie in IDPRs (Figure 10C3). The ELM resource has predicted 338 instances of 66 SLiMs within the protein, and after filtering out the structured domains, 178 instances of 51 disorder-based SLiMs were retained (Table 3). TheMoRF-associated SLiMs were identified and further described (Supplementary Table 2).

\subsection{Analysis of intrinsic disorder in transcription factors that are known JNK substrates}

\subsubsection{C-Jun (UniProt ID: P05412)}

Activator Protein -1 (AP - 1) complex is a term used for the dimers formed by proteins belonging to the JUN (JunB, JunD, c-Jun), FOS (c-Fos, FosB, Fra1, Fra2), ATF (ATF2, ATF3, B-ATF, JDP1 and JDP2) and Masculoaponeurotic fibrosarcoma (MAF) sub families and bZIP family. These proteins interact through the bZIP domain to form dimers [122,123]. c-Jun which is 331 amino acid long transcription factor is the most widely studied protein of the AP - 1 complex. The gene JUN which codes for this protein is intron less and has been mapped to chromosome 1 p32 - p31.Structure and function studies on this protein have shown that it can act as a homodimer or heterodimer and bind to DNA in order to regulate gene expression. This protein has two functionally important domains, a DNA binding domain (Residues: 227252) and the bZIP domain (Residues: 252-315). 
A $1.99 \AA$ r resolution XRD structure of c - Jun (Figure 11A1) is available on PDB (PDB ID: 5FV8, residues $277-308$ ), which indicates that only $9.67 \%$ of the protein structure is known. Figure $11 \mathrm{~A}$ represents the per - residue disorder propensity of $\mathrm{c}$ - Jun predicted by the six disorder predictors and their mean. As per the analysis, $\mathrm{c}$ - Jun belongs to the category of highly disordered proteins due to its PPID Mean Score of $57.10 \%$ (Table 2). This disorder is distributed in the form of three IDPRs, two of which are short and located at the $\mathrm{N}$ and $\mathrm{C}$ terminals of the protein. The third IDPR lies in the middle of the protein and houses

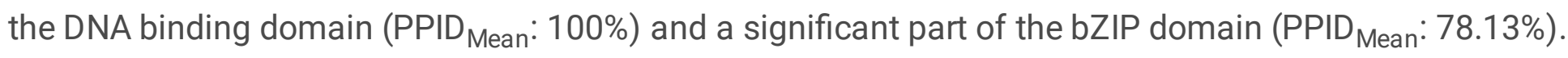

According to the result from $D^{2} P^{2}$ (Figure 11A2), $c$ - Jun contains 8 MoRF regions out of which 5 are embedded in the long IDPR. These predicted MoRF regions constitute $48.04 \%$ of the protein length (Table 3). c - Jun contains 18 phosphorylation sites, out of which 8 are disordered, two ubiquitylation sites, out of which one is disordered, two disordered sumoylation sites and three disordered acetylation sites (Figure 11A2). The ELM resource predicts that the protein has $51 \mathrm{SLiMs}$ and their 137 instances. After globular domain, structural and context filtering, only 47 SLiMs and their 103 instances are retained. The Supplementary Table 2 contains a detailed description and classification of SLiMs which are associated with MoRF regions.

\subsubsection{Global overview of intrinsic disorder in the other 29 TFs that are known as JNK substrates}

The PPID ${ }_{\text {Mean }}$ scores for the 29 transcription factors (Supplementary Table 2), the 2D disorder plot (Figure 12) and the PPID based classification method described before led us to conclude that none of these known JNK substrates are highly ordered. Highest level of intrinsic disorder was observed in the

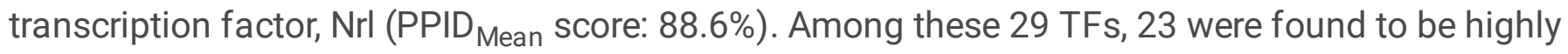
disordered; while, only 6 were identified as moderately disordered proteins.

\section{Concluding Remarks}

The JNK signaling cascade is a MAPK pathway that mediates response of eukaryotic cells to a wide range of abiotic and biotic stress conditions and stimuli. This pathway impacts gene expression and cell death/survival pathways. Oxidative stress is one such stimuli that occurs when the pro-oxidant/antioxidant balance of the cell is disturbed and results in activation of the JNK signaling cascade. Although the JNK signaling cascade has been under study for more than20 years, our studyrepresentsthe first systematic analysis of the abundance and functional roles of intrinsic disorder in proteins involved in this pathway. In this study, we have focused on the 18 proteins that are involved in the oxidative stressactivated extrinsic JNK signaling cascade.

As per our analysis, all proteins of the JNK signaling cascade possess significant levels of intrinsic disorder. Our findings align with the known fact that IDPRs are found in abundance in proteins involved in various cellular pathways, where they enhance and propagate signaling. IDPRs are also known to play 
important roles in assembly of signalosome complexes, and according to our analysis, the scaffold proteins (JIP1, JIP2, and JIP3), which are important for holding the JNK signalosome complex together, have the highest PPID values. The dual specificity phosphatases (MKP5 and MKP7), transcription factor c-Jun, and regulatory protein 14-3-3 3 have also shown high levels of intrinsic disorder. The MAP3Ks (ASK1 and ASK2), MAP2Ks (MKK4 and MKK7), MAPK (JNK1, JNK2 and JNK3) of the JNK signaling cascade and the proteins thioredoxin, TRAF2, TRAF6, and MKP1 were predicted to be moderately disordered. Furthermore, the highest percentage of disorder associated binding sites or MoRFs were also found in the scaffold proteins JIP1 and JIP2, as well as in the transcription factor c-Jun. This shows that the flexible nature of IDPRs may facilitate the capability of proteins to interact with multiple binding partners, which is important for signaling. For functional characterization of the predicted disordered regions, we identified the disorder associated functional motifs or SLiMs and further classified them into six classes based on their function. We also documented the PTM sites present in the proteins of the JNK signaling cascade and observed that a large number of PTM sites are embedded into the disordered regions.

High levels of oxidative stress have often been associated with various pathological conditions, such as neurodegeneration, diabetes, cancer, cardiovascular diseases, and immune system associated disorders, where they activate the JNK signaling cascade. As IDPRs constitute a noticeable (and non-negligible) fraction of the proteins involved in this pathway, further experimental characterization of these regions is extremely crucial to identify novel therapeutic targets to curb oxidative stress associated pathogenesis in disease conditions.

\section{Abbreviations}

$A \beta$ Amyloid $\beta$

AD Alzheimer's disease

AIDS Acquired Immunodeficiency syndrome

ALS Amyotrophic lateral sclerosis

AP1 Activator protein - 1

ASK Apoptosis regulating kinase

ATF2 Cyclic AMP-dependent transcription factor ATF-2

$D^{2} P^{2}$ Database of Disordered Protein predictions

DSP Dual Specificity phosphatases

ELM Eukaryotic Linear Motif 
ERK Extracellular signal - regulated kinases

IDP Intrinsically disordered protein

IDPR Intrinsically disordered protein region

JIP JNK interacting protein

JNK c-Jun N-terminal Kinase

MAP3K Mitogen Activated Kinase kinase kinase

MAP2K Mitogen Activated Protein Kinase kinase

MAPK Mitogen Activated Protein Kinase

MEF Mouse embryonic fibroblast

MKP Mitogen Activated Kinase phosphatases

MoRF Molecular recognition feature

NFATC3 Nuclear factor of activated T-cells, cytoplasmic 3

NLR NOD like receptor

NOD Nucleotide binding oligomerization domain

PD Parkinson's disease

PDB Protein Data Bank

PONDR Predictor of Natural Disordered Regions

PPID Predicted percentage of Intrinsic disorder

PTB Phosphotyrosine binding domain

PTM Posttranslational Modification

RLR RIG - I like receptor

ROS Reactive Oxygen Species

SAPK Stress Activated protein kinase

SLiM Short Linear Motif 
TLR Toll like receptor

TF Transcription factor

TNF-R Tumor necrosis factor receptor

TRAF Tumor necrosis factor receptor (TNF-R) associated factor

Txn Thioredoxin

\section{Declarations}

Data availability: Data are available in the Supplement.

\section{Compliance with ethical standards}

Conflict of interest: The authors declare no conflicts of interests.

Author Contributions: RG and VNU: conception and design, interpretation of data, writing, and review of the manuscript, and study supervision. BRG: acquisition and interpretation of data. BRG and KG: writing and review of the manuscript.

\section{Funding}

This research was funded by the Department of Biotechnology (DBT), India. BG is thankful to Ministry of Human Resource Development (MHRD) for funding. KG was supported by grant from DBT, India (BT/PR16871/NER/95/329/2015) to RG. VNU and RG are thankful to MHRD-SPARC (SPARC/20182019/P37/SL).

\section{Acknowledgments}

Authors would like to thank IIT Mandi for all the facilities.

\section{References}

[1] R.J. Davis, Signal transduction by the JNK group of MAP kinases, Cell. 103 (2000) 239-252. https://doi.org/10.1016/S0092-8674(00)00116-1.

[2] C.R. Weston, R.J. Davis, The JNK signal transduction pathway, Curr. Opin. Genet. Dev. 12 (2002) 1421. https://doi.org/10.1016/S0959-437X(01)00258-1.

[3] J. Cui, M. Zhang, Y.Q. Zhang, Z.H. Xu, JNK pathway: Diseases and therapeutic potential, Acta Pharmacol. Sin. 28 (2007) 601-608. https://doi.org/10.1111/j.1745-7254.2007.00579.x. 
[4] A. Zeke, M. Misheva, A. Reményi, M.A. Bogoyevitch, JNK Signaling: Regulation and Functions Based on Complex Protein-Protein Partnerships, Microbiol. Mol. Biol. Rev. 80 (2016) 793-835. https://doi.org/10.1128/mmbr.00043-14.

[5] E.A. Willoughby, G.R. Perkins, M.K. Collins, A.J. Whitmarsh, The JNK-interacting protein-1 scaffold protein targets MAPK phosphatase-7 to dephosphorylate JNK, J. Biol. Chem. 278 (2003) 10731-10736. https://doi.org/10.1074/jbc.M207324200.

[6] B.J. Pulverer, J.M. Kyriakis, J. Avruch, E. Nikolakaki, J.R. Woodgett, Phosphorylation of c-jun mediated by MAP kinases, Nature. 353 (1991) 670-674. https://doi.org/10.1038/353670a0.

[7] D.N. Dhanasekaran, E.P. Reddy, JNK signaling in apoptosis, Oncogene. 27 (2008) 6245-6251. https://doi.org/10.1038/onc.2008.301.

[8] Y. Xia, C. Makris, B. Su, E. Li, J. Yang, G.R. Nemerow, M. Karin, MEK kinase 1 is critically required for CJun N-terminal kinase activation by proinflammatory stimuli and growth factor-induced cell migration, Proc. Natl. Acad. Sci. U. S. A. 97 (2000) 5243-5248. https://doi.org/10.1073/pnas.97.10.5243.

[9] K.J. Barnham, C.L. Masters, A.I. Bush, Neurodegenerative diseases and oxidatives stress, Nat. Rev. Drug Discov. 3 (2004) 205-214. https://doi.org/10.1038/nrd1330.

[10] V. Sosa, T. Moliné, R. Somoza, R. Paciucci, H. Kondoh, M.E. LLeonart, Oxidative stress and cancer: An overview, Ageing Res. Rev. 12 (2013) 376-390. https://doi.org/10.1016/j.arr.2012.10.004.

[11] J.F. Turrens, A. Boveris, Generation of superoxide anion by the NADH dehydrogenase of bovine heart mitochondria., Biochem. J. 191 (1980) 421-427. https://doi.org/10.1042/bj1910421.

[12] K. Sinha, J. Das, P.B. Pal, P.C. Sil, Oxidative stress: The mitochondria-dependent and mitochondriaindependent pathways of apoptosis, Arch. Toxicol. 87 (2013) 1157-1180.

https://doi.org/10.1007/s00204-013-1034-4.

[13] M. Saitoh, H. Nishitoh, M. Fujii, K. Takeda, K. Tobiume, Y. Sawada, M. Kawabata, K. Miyazono, H. Ichijo, Mammalian thioredoxin is a direct inhibitor of apoptosis signal-regulating kinase (ASK) 1, EMBO J. 17 (1998) 2596-2606.

[14] O. Singh, A. Shillings, P. Craggs, I. Wall, P. Rowland, T. Skarzynski, C.I. Hobbs, P. Hardwick, R. Tanner, M. Blunt, D.R. Witty, K.J. Smith, Crystal structures of ASK1-inhibtor complexes provide a platform for structure-based drug design, Protein Sci. 22 (2013) 1071-1077. https://doi.org/10.1002/pro.2298.

[15] J. Yasuda, A.J. Whitmarsh, J. Cavanagh, M. Sharma, R.J. Davis, The JIP Group of Mitogen-Activated Protein Kinase Scaffold Proteins, Mol. Cell. Biol. 19 (1999) 7245-7254.

https://doi.org/10.1128/mcb.19.10.7245. 
[16] B. Dérijard, M. Hibi, I.H. Wu, T. Barrett, B. Su, T. Deng, M. Karin, R.J. Davis, JNK1: A protein kinase stimulated by UV light and Ha-Ras that binds and phosphorylates the c-Jun activation domain, Cell. 76 (1994) 1025-1037. https://doi.org/10.1016/0092-8674(94)90380-8.

[17] S. Gupta, D. Campbell, B. Dérijard, R.J. Davis, Transcription factor ATF2 regulation by the JNK signal transduction pathway, Science (80-. ). 267 (1995) 389-393. https://doi.org/10.1126/science.7824938.

[18] C.R. Weston, R.J. Davis, The JNK signal transduction pathway, Curr. Opin. Cell Biol. 19 (2007) 142149. https://doi.org/10.1016/j.ceb.2007.02.001.

[19] R. Van Der Lee, M. Buljan, B. Lang, R.J. Weatheritt, G.W. Daughdrill, A.K. Dunker, M. Fuxreiter, J. Gough, J. Gsponer, D.T. Jones, P.M. Kim, R.W. Kriwacki, C.J. Oldfield, R. V. Pappu, P. Tompa, V.N. Uversky, P.E. Wright, M.M. Babu, Classification of intrinsically disordered regions and proteins, Chem. Rev. 114 (2014) 6589-6631. https://doi.org/10.1021/cr400525m.

[20] T. Chouard, Structural biology: Breaking the protein rules, Nature. 471 (2011) 151-153. https://doi.org/10.1038/471151a.

[21] P.E. Wright, H.J. Dyson, Intrinsically unstructured proteins: Re-assessing the protein structure-function paradigm, J. Mol. Biol. 293 (1999) 321-331. https://doi.org/10.1006/jmbi.1999.3110.

[22] P.E. Wright, H.J. Dyson, Intrinsically disordered proteins in cellular signalling and regulation, Nat. Rev. Mol. Cell Biol. 16 (2015) 18-29. https://doi.org/10.1038/nrm3920.

[23] A. Katuwawala, Z. Peng, J. Yang, L. Kurgan, Computational Prediction of MoRFs, Short Disorder-toorder Transitioning Protein Binding Regions, Comput. Struct. Biotechnol. J. 17 (2019) 454-462. https://doi.org/10.1016/j.csbj.2019.03.013.

[24] V.N. Uversky, C.J. Oldfield, A.K. Dunker, Intrinsically Disordered Proteins in Human Diseases: Introducing the D 2 Concept , Annu. Rev. Biophys. 37 (2008) 215-246. https://doi.org/10.1146/annurev.biophys.37.032807.125924.

[25] A. et al. Bateman, UniProt: The universal protein knowledgebase, Nucleic Acids Res. 45 (2017) D158D169. https://doi.org/10.1093/nar/gkw1099.

[26] R. P, O. Z, L. X, G. EC, B. CJ, D. AK, Sequence Complexity of Disordered Protein, Proteins. 42 (2001). https://doi.org/10.1002/1097-0134(20010101)42:1<38::AID-PROT50>3.0.C0;2-3.

[27] Peng Kang, R. Predrag, V. Slobodan, D.A. Keith, O. Zoran, Length-dependent Prediction of Protein Intrinsic Disorder, BMC Bioinformatics. (2006). https://pubmed.ncbi.nlm.nih.gov/16618368/ (accessed June 22, 2020).

[28] K. Peng, S. Vucetic, P. Radivojac, C.J. Brown, A.K. Dunker, Z. Obradovic, Optimizing long intrinsic disorder predictors with protein evolutionary information, J. Bioinform. Comput. Biol. 3 (2005) 35-60. 
https://doi.org/10.1142/S0219720005000886.

[29] S.I. Dosztányi Zsuzsanna, Csizmok Veronika, Tompa Peter, IUPred: Web Server for the Prediction of Intrinsically Unstructured Regions of Proteins Based on Estimated Energy Content, Bioinformatics. (2005). https://pubmed.ncbi.nlm.nih.gov/15955779/ (accessed June 22, 2020).

[30] B. Xue, R.L. Dunbrack, R.W. Williams, A.K. Dunker, V.N. Uversky, PONDR-FIT: A meta-predictor of intrinsically disordered amino acids, Biochim. Biophys. Acta - Proteins Proteomics. 1804 (2010) 9961010. https://doi.org/10.1016/j.bbapap.2010.01.011.

[31] O.M. E, R. Pedro, I. Takashi, G. Mohamed, M.M. J, X. Bin, D. Zsuzsanna, U.V. N, O. Zoran, K. Lukasz, D.A. Keith, G. Julian, D²P2: Database of Disordered Protein Predictions, Nucleic Acids Res. (2013). https://pubmed.ncbi.nlm.nih.gov/23203878/ (accessed June 23, 2020).

[32] M. Gouw, H. Sámano-Sánchez, K. Van Roey, F. Diella, T.J. Gibson, H. Dinkel, Exploring short linear motifs using the ELM database and tools, Curr. Protoc. Bioinforma. 2017 (2017) 8.22.1-8.22.35. https://doi.org/10.1002/cpbi.26.

[33] D. Szklarczyk, A.L. Gable, D. Lyon, A. Junge, S. Wyder, J. Huerta-Cepas, M. Simonovic, N.T. Doncheva, J.H. Morris, P. Bork, L.J. Jensen, C. von Mering, C. Von Mering, STRING v11: Protein-Protein Association Networks With Increased Coverage, Supporting Functional Discovery in Genome-Wide Experimental Datasets, Nucleic Acids Res. 47 (2019) D607-D613. https://pubmed.ncbi.nlm.nih.gov/30476243/ (accessed June 23, 2020).

[34] E.F. Pettersen, T.D. Goddard, C.C. Huang, G.S. Couch, D.M. Greenblatt, E.C. Meng, T.E. Ferrin, UCSF Chimera - A visualization system for exploratory research and analysis, J. Comput. Chem. 25 (2004) 1605-1612. https://doi.org/10.1002/jcc.20084.

[35] Y. Asaoka, H. Nishina, Diverse physiological functions of MKK4 and MKK7 during early embryogenesis, J. Biochem. 148 (2010) 393-401. https://doi.org/10.1093/jb/mvq098.

[36] T. Fujisawa, K. Takeda, H. Ichijo, ASK family proteins in stress response and disease, in: Mol. Biotechnol., Mol Biotechnol, 2007: pp. 13-18. https://doi.org/10.1007/s12033-007-0053-x.

[37] K. Takeda, R. Shimozono, T. Noguchi, T. Umeda, Y. Morimoto, I. Naguro, K. Tobiume, M. Saitoh, A. Matsuzawa, H. Ichijo, Apoptosis signal-regulating kinase (ASK) 2 functions as a mitogen-activated protein kinase kinase kinase in a heteromeric complex with ASK1, J. Biol. Chem. 282 (2007) 7522-7531. https://doi.org/10.1074/jbc.M607177200.

[38] M. Saitoh, H. Nishitoh, M. Fujii, K. Takeda, K. Tobiume, Y. Sawada, M. Kawabata, K. Miyazono, H. Ichijo, Mammalian thioredoxin is a direct inhibitor of apoptosis signal-regulating kinase (ASK) 1, EMBO J. 17 (1998) 2596-2606. https://doi.org/10.1093/emboj/17.9.2596. 
[39] H. Liu, H. Nishitoh, H. Ichijo, J.M. Kyriakis, Activation of Apoptosis Signal-Regulating Kinase 1 (ASK1) by Tumor Necrosis Factor Receptor-Associated Factor 2 Requires Prior Dissociation of the ASK1 Inhibitor Thioredoxin, Mol. Cell. Biol. 20 (2000) 2198-2208. https://doi.org/10.1128/mcb.20.6.2198-2208.2000.

[40] L.M. Cockrell, M.C. Puckett, E.H. Goldman, F.R. Khuri, H. Fu, Dual engagement of 14-3-3 proteins controls signal relay from ASK2 to the ASK1 signalosome, Oncogene. 29 (2010) 822-830. https://doi.org/10.1038/onc.2009.382.

[41] T. Noguchi, K. Takeda, A. Matsuzawa, K. Saegusa, H. Nakano, J. Gohda, J.I. Inoue, H. Ichijo, Recruitment of tumor necrosis factor receptor-associated factor family proteins to apoptosis signalregulating kinase 1 signalosome is essential for oxidative stress-induced cell death, J. Biol. Chem. 280 (2005) 37033-37040. https://doi.org/10.1074/jbc.M506771200.

[42] G. Fujino, T. Noguchi, A. Matsuzawa, S. Yamauchi, M. Saitoh, K. Takeda, H. Ichijo, Thioredoxin and TRAF Family Proteins Regulate Reactive Oxygen Species-Dependent Activation of ASK1 through Reciprocal Modulation of the N-Terminal Homophilic Interaction of ASK1, Mol. Cell. Biol. 27 (2007) 81528163. https://doi.org/10.1128/mcb.00227-07.

[43] C. Tournier, C. Dong, T.K. Turner, S.N. Jones, R.A. Flavell, R.J. Davis, MKK7 is an essential component of the JNK signal transduction pathway activated by proinflammatory cytokines, Genes Dev. 15 (2001) 1419-1426. https://doi.org/10.1101/gad.888501.

[44] J. Liu, A. Lin, Role of JNK activation in apoptosis: A double-edged sword, Cell Res. 15 (2005) 36-42. https://doi.org/10.1038/sj.cr.7290262.

[45] A.J. Whitmarsh, J. Cavanagh, C. Tournier, J. Yasuda, R.J. Davis, A mammalian scaffold complex that selectively mediates MAP kinase activation, Science (80-. ). 281 (1998) 1671-1674. https://doi.org/10.1126/science.281.5383.1671.

[46] O. Bermudez, G. Pagès, C. Gimond, The dual-specificity MAP kinase phosphatases: Critical roles in development and cancer, Am. J. Physiol. - Cell Physiol. 299 (2010). https://doi.org/10.1152/ajpcell.00347.2009.

[47] Q. Meng, Y. Xia, c-Jun, at the crossroad of the signaling network, Protein Cell. 2 (2011) 889-898. https://doi.org/10.1007/s13238-011-1113-3.

[48] K. Rajagopalan, S.M. Mooney, N. Parekh, R.H. Getzenberg, P. Kulkarni, A majority of the cancer/testis antigens are intrinsically disordered proteins, J. Cell. Biochem. 112 (2011) 3256-3267. https://doi.org/10.1002/jcb.23252.

[49] K. Gadhave, B.R. Gehi, P. Kumar, B. Xue, V.N. Uversky, R. Giri, The dark side of Alzheimer's disease: unstructured biology of proteins from the amyloid cascade signaling pathway, Cell. Mol. Life Sci. (2020). https://doi.org/10.1007/s00018-019-03414-9. 
[50] Z. Dosztányi, B. Mészáros, I. Simon, ANCHOR: Web server for predicting protein binding regions in disordered proteins, Bioinformatics. 25 (2009) 2745-2746.

https://doi.org/10.1093/bioinformatics/btp518.

[51] S. Shiizaki, I. Naguro, H. Ichijo, Activation mechanisms of ASK1 in response to various stresses and its significance in intracellular signaling, Adv. Biol. Regul. 53 (2013) 135-144.

https://doi.org/10.1016/j.jbior.2012.09.006.

[52] G. Bunkoczi, E. Salah, P. Filippakopoulos, O. Fedorov, S. Müller, F. Sobott, S.A. Parker, H. Zhang, W. Min, B.E. Turk, S. Knapp, Structural and Functional Characterization of the Human Protein Kinase ASK1, Structure. 15 (2007) 1215-1226. https://doi.org/10.1016/j.str.2007.08.011.

[53] H. Ichijo, E. Nishida, K. Irie, P. Ten Dijke, M. Saitoh, T. Moriguchi, M. Takagi, K. Matsumoto, K. Miyazono, Y. Gotoh, Induction of apoptosis by ASK1, a mammalian MAPKKK that activates SAPK/JNK and p38 signaling pathways, Science (80-. ). 275 (1997) 90-94.

https://doi.org/10.1126/science.275.5296.90.

[54] Y.R. Chen, X. Wang, D. Templeton, R.J. Davis, T.H. Tan, The role of c-Jun N-terminal kinase (JNK) in apoptosis induced by ultraviolet $\mathrm{C}$ and $\mathrm{y}$ radiation. Duration of JNK activation may determine cell death and proliferation, J. Biol. Chem. 271 (1996) 31929-31936. https://doi.org/10.1074/jbc.271.50.31929.

[55] P.H. McDonald, beta -Arrestin 2: A Receptor-Regulated MAPK Scaffold for the Activation of JNK3, Science (80-. ). 290 (2000) 1574-1577. https://doi.org/10.1126/science.290.5496.1574.

[56] Y. Liu, W. Min, Thioredoxin promotes ASK1 ubiquitination and degradation to inhibit ASK1-mediated apoptosis in a redox activity-independent manner, Circ. Res. 90 (2002) 1259-1266.

https://doi.org/10.1161/01.RES.0000022160.64355.62.

[57] K. Tobiume, M. Saitoh, H. Ichijo, Activation of apoptosis signal-regulating Kinase 1 by the stressinduced activating phosphorylation of pre-formed oligomer, J. Cell. Physiol. 191 (2002) 95-104. https://doi.org/10.1002/jcp.10080.

[58] Y. Hashimoto, T. Niikura, T. Chiba, E. Tsukamoto, H. Kadowaki, H. Nishitoh, Y. Yamagishi, M. Ishizaka, M. Yamada, M. Nawa, K. Terashita, S. Aiso, H. Ichijo, I. Nishimoto, The cytoplasmic domain of Alzheimer's amyloid- $\beta$ protein precursor causes sustained apoptosis signal-regulating kinase 1/c-Jun NH 2-terminal kinase-mediated neurotoxic signal via dimerization, J. Pharmacol. Exp. Ther. 306 (2003) 889-902. https://doi.org/10.1124/jpet.103.051383.

[59] H. Kadowaki, H. Nishitoh, F. Urano, C. Sadamitsu, A. Matsuzawa, K. Takeda, H. Masutani, J. Yodoi, Y. Urano, T. Nagano, H. Ichijo, Amyloid $\beta$ induces neuronal cell death through ROS-mediated ASK1 activation, Cell Death Differ. 12 (2005) 19-24. https://doi.org/10.1038/sj.cdd.4401528. 
[60] J. Zhang, E.S. Park, H.J. Park, R. Yan, M. Grudniewska, X. Zhang, S. Oh, X. Yang, J. Baum, M.M. Mouradian, Apoptosis signal regulating kinase 1 deletion mitigates a-synuclein pre-formed fibril propagation in mice, Neurobiol. Aging. 85 (2020) 49-57.

https://doi.org/10.1016/j.neurobiolaging.2019.09.012.

[61] J.F. Weijman, A. Kumar, S.A. Jamieson, C.M. King, T.T. Caradoc-Davies, E.C. Ledgerwood, J.M. Murphy, P.D. Mace, Structural basis of autoregulatory scaffolding by apoptosis signal-regulating kinase 1, Proc. Natl. Acad. Sci. U. S. A. 114 (2017) E2096-E2105. https://doi.org/10.1073/pnas.1620813114.

[62] O. Petrvalska, D. Kosek, Z. Kukacka, Z. Tosner, P. Man, J. Vecer, P. Herman, V. Obsilova, T. Obsil, Structural insight into the 14-3-3 protein-dependent inhibition of protein kinase ASK1 (apoptosis signalregulating kinase 1), J. Biol. Chem. 291 (2016) 20753-20765. https://doi.org/10.1074/jbc.M116.724310.

[63] E. Ortner, K. Moelling, Heteromeric complex formation of ASK2 and ASK1 regulates stress-induced signaling, Biochem. Biophys. Res. Commun. 362 (2007) 454-459.

https://doi.org/10.1016/j.bbrc.2007.08.006.

[64] T. Iriyama, K. Takeda, H. Nakamura, Y. Morimoto, T. Kuroiwa, J. Mizukami, T. Umeda, T. Noguchi, I. Naguro, H. Nishitoh, K. Saegusa, K. Tobiume, T. Homma, Y. Shimada, H. Tsuda, S. Aiko, I. Imoto, J. Inazawa, K. Chida, Y. Kamei, S. Kozuma, Y. Taketani, A. Matsuzawa, H. Ichijo, ASK1 and ASK2 differentially regulate the counteracting roles of apoptosis and inflammation in tumorigenesis, EMBO J. 28 (2009) 843-853. https://doi.org/10.1038/emboj.2009.32.

[65] K. Fritz-Wolf, S. Kehr, M. Stumpf, S. Rahlfs, K. Becker, Crystal structure of the human thioredoxin reductase-thioredoxin complex, Nat. Commun. 2 (2011). https://doi.org/10.1038/ncomms1382.

[66] G. Spyrou, E. Enmark, A. Miranda-Vizuete, J.Å. Gustafsson, Cloning and expression of a novel mammalian thioredoxin, J. Biol. Chem. 272 (1997) 2936-2941. https://doi.org/10.1074/jbc.272.5.2936.

[67] R. Zhang, R. Al-Lamki, L. Bai, J.W. Streb, J.M. Miano, J. Bradley, W. Min, Thioredoxin-2 inhibits mitochondria-located ASK1-mediated apoptosis in a JNK-independent manner, Circ. Res. 94 (2004) 1483-1491. https://doi.org/10.1161/01.RES.0000130525.37646.a7.

[68] K.F. Tonissen, J.R.E. Wells, Isolation and characterization of human thioredoxin-encoding genes, Gene. 102 (1991) 221-228. https://doi.org/10.1016/0378-1119(91)90081-L.

[69] A. Holmgren, Thioredoxin, Annu. Rev. Biochem. 54 (1985) 237-271.

https://doi.org/10.1146/annurev.bi.54.070185.001321.

[70] G. Powis, W.R. Montfort, Properties and biological activities of thioredoxins, Annu. Rev. Biophys. Biomol. Struct. 30 (2001) 421-455. https://doi.org/10.1146/annurev.biophys.30.1.421.

[71] H. Fu, R.R. Subramanian, S.C. Masters, 14-3-3 Proteins: Structure, Function, and Regulation, Annu. Rev. Pharmacol. Toxicol. 40 (2000) 617-647. https://doi.org/10.1146/annurev.pharmtox.40.1.617. 
[72] K. Pennington, T. Chan, M. Torres, J. Andersen, The dynamic and stress-adaptive signaling hub of 143-3: emerging mechanisms of regulation and context-dependent protein-protein interactions, Oncogene. 37 (2018) 5587-5604. https://doi.org/10.1038/s41388-018-0348-3.

[73] E.H. Goldman, L. Chen, H. Fu, Activation of Apoptosis Signal-regulating Kinase 1 by Reactive Oxygen Species through Dephosphorylation at Serine 967 and 14-3-3 Dissociation, J. Biol. Chem. 279 (2004) 10442-10449. https://doi.org/10.1074/jbc.M311129200.

[74] K. Kaneko, N.S. Hachiya, The alternative role of 14-3-3 zeta as a sweeper of misfolded proteins in disease conditions, Med. Hypotheses. 67 (2006) 169-171. https://doi.org/10.1016/j.mehy.2006.01.019.

[75] Z.Y. Foote Molly, 14-3-3 proteins in neurological disorders, Int J Biochem Mol Biol . 3 (2012) 152-164. www.ijbmb.org (accessed June 23, 2020).

[76] K. Rittinger, J. Budman, J. Xu, S. Volinia, L.C. Cantley, S.J. Smerdon, S.J. Gamblin, M.B. Yaffe, Structural analysis of 14-3-3 phosphopeptide complexes identifies a dual role for the nuclear export signal of 14-3-3 in ligand binding, Mol. Cell. 4 (1999) 153-166. https://doi.org/10.1016/S10972765(00)80363-9.

[77] P. Xie, TRAF molecules in cell signaling and in human diseases, J. Mol. Signal. 8 (2013). https://doi.org/10.1186/1750-2187-8-7.

[78] H.H. Park, Structure of TRAF Family: Current Understanding of Receptor Recognition, Front. Immunol. 9 (2018) 1999. https://doi.org/10.3389/fimmu.2018.01999.

[79] J.R. Bradley, J.S. Pober, Tumor necrosis factor receptor-associated factors (TRAFs), Oncogene. 20 (2001) 6482-6491. https://doi.org/10.1038/sj.onc.1204788.

[80] Q. Yin, B. Lamothe, B.G. Darnay, H. Wu, Structural basis for the lack of E2 interaction in the RING domain of TRAF2, Biochemistry. 48 (2009) 10558-10567. https://doi.org/10.1021/bi901462e.

[81] S.M. McWhirter, S.S. Pullen, J.M. Holton, J.J. Crute, M.R. Kehry, T. Alber, Crystallographic analysis of CD40 recognition and signaling by human TRAF2, Proc. Natl. Acad. Sci. U. S. A. 96 (1999) 8408-8413. https://doi.org/10.1073/pnas.96.15.8408.

[82] C. Zheng, V. Kabaleeswaran, Y. Wang, G. Cheng, H. Wu, Crystal Structures of the TRAF2: ClAP2 and the TRAF1: TRAF2: clAP2 Complexes: Affinity, Specificity, and Regulation, Mol. Cell. 38 (2010) 101-113. https://doi.org/10.1016/j.molcel.2010.03.009.

[83] Q. Yin, S.C. Lin, B. Lamothe, M. Lu, Y.C. Lo, G. Hura, L. Zheng, R.L. Rich, A.D. Campos, D.G. Myszka, M.J. Lenardo, B.G. Darnay, H. Wu, E2 interaction and dimerization in the crystal structure of TRAF6, Nat. Struct. Mol. Biol. 16 (2009) 658-666. https://doi.org/10.1038/nsmb.1605. 
[84] K. Gadhave, P. Kumar, S.K. Kapuganti, V.N. Uversky, R. Giri, Unstructured Biology of Proteins from Ubiquitin-Proteasome System: Roles in Cancer and Neurodegenerative Diseases, Biomolecules. 10 (2020) 796. https://doi.org/10.3390/biom10050796.

[85] H. Ye, J.R. Arron, B. Lamothe, M. Cirilli, T. Kobayashi, N.K. Shevde, D. Segal, O.K. Dzivenu, M. Vologodskaia, M. Yim, K. Du, S. Singh, J.W. Pike, B.G. Darnay, Y. Choi, H. Wu, Distinct molecular mechanism for initiating TRAF6 signalling, Nature. 418 (2002) 443-447. https://doi.org/10.1038/nature00888.

[86] C. Widmann, S. Gibson, M.B. Jarpe, G.L. Johnson, Mitogen-activated protein kinase: Conservation of a three-kinase module from yeast to human, Physiol. Rev. 79 (1999) 143-180. https://doi.org/10.1152/physrev.1999.79.1.143.

[87] X. Wang, A. Destrument, C. Tournier, Physiological roles of MKK4 and MKK7: Insights from animal models, Biochim. Biophys. Acta - Mol. Cell Res. 1773 (2007) 1349-1357.

https://doi.org/10.1016/j.bbamcr.2006.10.016.

[88] J.G. Park, N. Aziz, J.Y. Cho, MKK7, the essential regulator of JNK signaling involved in cancer cell survival: a newly emerging anticancer therapeutic target, Ther. Adv. Med. Oncol. 11 (2019). https://doi.org/10.1177/1758835919875574.

[89] T. Matsumoto, T. Kinoshita, Y. Kirii, K. Yokota, K. Hamada, T. Tada, Crystal structures of MKK4 kinase domain reveal that substrate peptide binds to an allosteric site and induces an auto-inhibition state, Biochem. Biophys. Res. Commun. 400 (2010) 369-373. https://doi.org/10.1016/j.bbrc.2010.08.071.

[90] Y. Sogabe, T. Hashimoto, T. Matsumoto, Y. Kirii, M. Sawa, T. Kinoshita, A crucial role of Cys218 in configuring an unprecedented auto-inhibition form of MAP2K7, Biochem. Biophys. Res. Commun. 473 (2016) 476-481. https://doi.org/10.1016/j.bbrc.2016.03.036.

[91] A. Estaña, N. Sibille, E. Delaforge, M. Vaisset, J. Cortés, P. Bernadó, Realistic Ensemble Models of Intrinsically Disordered Proteins Using a Structure-Encoding Coil Database, Structure. 27 (2019) 381391.e2. https://doi.org/10.1016/j.str.2018.10.016.

[92] L. Resnick, M. Fennell, Targeting JNK3 for the treatment of neurodegenerative disorders, Drug Discov. Today. 9 (2004) 932-939. https://doi.org/10.1016/S1359-6446(04)03251-9.

[93] M. Raciti, L. V. Lotti, S. Valia, F.M. Pulcinelli, L. Di Renzo, JNK2 is activated during ER stress and promotes cell survival, Cell Death Dis. 3 (2012). https://doi.org/10.1038/cddis.2012.167.

[94] V. Aguirre, T. Uchida, L. Yenush, R. Davis, M.F. White, The c-Jun NH2-terminal kinase promotes insulin resistance during association with insulin receptor substrate-1 and phosphorylation of Ser307, J. Biol. Chem. 275 (2000) 9047-9054. https://doi.org/10.1074/jbc.275.12.9047. 
[95] A. Jaeschke, M.P. Czech, R.J. Davis, An essential role of the JIP1 scaffold protein for JNK activation in adipose tissue, Genes Dev. 18 (2004) 1976-1980. https://doi.org/10.1101/gad.1216504.

[96] A. Jaeschke, M. Rincón, B. Doran, J. Reilly, D. Neuberg, D.L. Greiner, L.D. Shultz, A.A. Rossini, R.A. Flavell, R.J. Davis, Disruption of the Jnk2 (Mapk9) gene reduces destructive insulitis and diabetes in a mouse model of type 1 diabetes, Proc. Natl. Acad. Sci. U. S. A. 102 (2005) 6931-6935. https://doi.org/10.1073/pnas.0502143102.

[97] H. Kaneto, Y. Nakatani, T. Miyatsuka, D. Kawamori, T.A. Matsuoka, M. Matsuhisa, Y. Kajimoto, H. Ichijo, Y. Yamasaki, M. Hori, Possible novel therapy for diabetes with cell-permeable JNK-inhibitory peptide, Nat. Med. 10 (2004) 1128-1132. https://doi.org/10.1038/nm1111.

[98] Á. Garai, A. Zeke, G. Gógl, I. Töro, F. Fördos, H. Blankenburg, T. Bárkai, J. Varga, A. Alexa, D. Emig, M. Albrecht, A. Reményi, Specificity of linear motifs that bind to a common mitogen-activated protein kinase docking groove, Sci. Signal. 5 (2012). https://doi.org/10.1126/scisignal.2003004.

[99] D. Shaw, S.M. Wang, A.G. Villaseñor, S. Tsing, D. Walter, M.F. Browner, J. Barnett, A. Kuglstatter, The Crystal Structure of JNK2 Reveals Conformational Flexibility in the MAP Kinase Insert and Indicates Its Involvement in the Regulation of Catalytic Activity, J. Mol. Biol. 383 (2008) 885-893. https://doi.org/10.1016/j.jmb.2008.08.086.

[100] V.P. Krenitsky, M. Delgado, L. Nadolny, K. Sahasrabudhe, L. Ayala, S.S. Clareen, R. Hilgraf, R. Albers, A. Kois, K. Hughes, J. Wright, J. Nowakowski, E. Sudbeck, S. Ghosh, S. Bahmanyar, P. Chamberlain, J. Muir, B.E. Cathers, D. Giegel, L. Xu, M. Celeridad, M. Moghaddam, O. Khatsenko, P. Omholt, J. Katz, S. Pai, R. Fan, Y. Tang, M.A. Shirley, B. Benish, K. Blease, H. Raymon, S. Bhagwat, I. Henderson, A.G. Cole, B. Bennett, Y. Satoh, Aminopurine based JNK inhibitors for the prevention of ischemia reperfusion injury, Bioorganic Med. Chem. Lett. 22 (2012) 1427-1432. https://doi.org/10.1016/j.bmcl.2011.12.028.

[101] J.D. Laughlin, J.C. Nwachukwu, M. Figuera-Losada, L. Cherry, K.W. Nettles, P. V. Lograsso, Structural mechanisms of allostery and autoinhibition in JNK family kinases, Structure. 20 (2012) 2174-2184. https://doi.org/10.1016/j.str.2012.09.021.

[102] S. Pernigo, M.S. Chegkazi, Y.Y. Yip, C. Treacy, G. Glorani, K. Hansen, A. Politis, S. Bui, M.P. Dodding, R.A. Steiner, Structural basis for isoform-specific kinesin-1 recognition of Y-acidic cargo adaptors, Elife. 7 (2018). https://doi.org/10.7554/eLife.38362.

[103] A.J. Whitmarsh, C.Y. Kuan, N.J. Kennedy, N. Kelkar, T.F. Haydar, J.P. Mordes, M. Appel, A.A. Rossini, S.N. Jones, R.A. Flavell, P. Rakic, R.J. Davis, Requirement of the JIP1 scaffold protein for stress-induced JNK activation, Genes Dev. 15 (2001) 2421-2432. https://doi.org/10.1101/gad.922801.

[104] D. Meyer, A. Liu, B. Margolis, Interaction of c-Jun amino-terminal kinase interacting protein-1 with p190 rhoGEF and its localization in differentiated neurons, J. Biol. Chem. 274 (1999) 35113-35118. https://doi.org/10.1074/jbc.274.49.35113. 
[105] W. Stockinger, C. Brandes, D. Fasching, M. Hermann, M. Gotthardt, J. Herz, W.J. Schneider, J. Nimpf, The reelin receptor ApoER2 recruits JNK-interacting proteins-1 and -2, J. Biol. Chem. 275 (2000) 2562525632. https://doi.org/10.1074/jbc.M004119200.

[106] M. Gotthardt, M. Trommsdorff, M.F. Nevitt, J. Shelton, J.A. Richardson, W. Stockinger, J. Nimpf, J. Herz, Interactions of the low density lipoprotein receptor gene family with cytosolic adaptor and scaffold proteins suggest diverse biological functions in cellular communication and signal transduction, J. Biol. Chem. 275 (2000) 25616-25624. https://doi.org/10.1074/jbc.M000955200.

[107] K.J. Verhey, D. Meyer, R. Deehan, J. Blenis, B.J. Schnapp, T.A. Rapoport, B. Margolis, Cargo of kinesin identified as JIP scaffolding proteins and associated signaling molecules, J. Cell Biol. 152 (2001) 959970. https://doi.org/10.1083/jcb.152.5.959.

[108] M.M. Fu, E.L.F. Holzbaur, JIP1 regulates the directionality of APP axonal transport by coordinating kinesin and dynein motors, J. Cell Biol. 202 (2013) 495-508. https://doi.org/10.1083/jcb.201302078.

[109] A.J. Whitmarsh, The JIP family of MAPK scaffold proteins, Biochem. Soc. Trans. 34 (2006) 828832. https://doi.org/10.1042/BST0340828.

[110] H. Taru, K.I. lijima, M. Hase, Y. Kirino, Y. Yagi, T. Suzuki, Interaction of Alzheimer's $\beta$-amyloid precursor family proteins with scaffold proteins of the JNK signaling cascade, J. Biol. Chem. 277 (2002) 20070-20078. https://doi.org/10.1074/jbc.M108372200.

[111] N. Kelkar, S. Gupta, M. Dickens, R.J. Davis, Interaction of a Mitogen-Activated Protein Kinase Signaling Module with the Neuronal Protein JIP3, Mol. Cell. Biol. 20 (2000) 1030-1043. https://doi.org/10.1128/mcb.20.3.1030-1043.2000.

[112] T. Sun, N. Yu, L.K. Zhai, N. Li, C. Zhang, L. Zhou, Z. Huang, X.Y. Jiang, Y. Shen, Z.Y. Chen, C-Jun NH2terminal kinase (JNK)-interacting protein-3 (JIP3) regulates neuronal axon elongation in a kinesin- And JNK-dependent manner, J. Biol. Chem. 288 (2013) 14531-14543.

https://doi.org/10.1074/jbc.M113.464453.

[113] P. Llinas, M. Chenon, T.Q. Nguyen, C. Moreira, A. De Régibus, A. Coquard, M.J. Ramos, R. Guérois, P.A. Fernandes, J. Ménétrey, Structure of a truncated form of leucine zipper II of JIP3 reveals an unexpected antiparallel coiled-coil arrangement, Acta Crystallogr. Sect. Struct. Biol. Commun. 72 (2016) 198-206. https://doi.org/10.1107/S2053230X16001576.

[114] D.M. Owens, S.M. Keyse, Differential regulation of MAP kinase signalling by dual-specificity protein phosphatases, Oncogene. 26 (2007) 3203-3213. https://doi.org/10.1038/sj.onc.1210412.

[115] K.I. Patterson, T. Brummer, P.M. O'Brien, R.J. Daly, Dual-specificity phosphatases: Critical regulators with diverse cellular targets, Biochem. J. 418 (2009) 475-489. https://doi.org/10.1042/BJ20082234. 
[116] X. Liu, C.S. Zhang, C. Lu, S.C. Lin, J.W. Wu, Z.X. Wang, A conserved motif in JNK/p38-specific MAPK phosphatases as a determinant for JNK1 recognition and inactivation, Nat. Commun. 7 (2016). https://doi.org/10.1038/ncomms10879.

[117] Y. Son, Y.-K. Cheong, N.-H. Kim, H.-T. Chung, D.G. Kang, H.-O. Pae, Mitogen-Activated Protein Kinases and Reactive Oxygen Species: How Can ROS Activate MAPK Pathways?, J. Signal Transduct. 2011 (2011). https://doi.org/10.1155/2011/792639.

[118] C.H. Teng, W.N. Huang, T.C. Meng, Several dual specificity phosphatases coordinate to control the magnitude and duration of JNK activation in signaling response to oxidative stress, J. Biol. Chem. 282 (2007) 28395-28407. https://doi.org/10.1074/jbc.M705142200.

[119] J.Y. Zhou, Y. Liu, S.W. Gen, The role of mitogen-activated protein kinase phosphatase-1 in oxidative damage-induced cell death, Cancer Res. 66 (2006) 4888-4894. https://doi.org/10.1158/0008-5472.CAN05-4229.

[120] R. Gumpena, G.T. Lountos, D.S. Waugh, MBP-binding DARPins facilitate the crystallization of an MBP fusion protein, Acta Crystallogr. Sect. F Struct. Biol. Commun. 74 (2018) 549-557. https://doi.org/10.1107/S2053230X18009901.

[121] X. Tao, L. Tong, Crystal structure of the MAP kinase binding domain and the catalytic domain of human MKP5, Protein Sci. 16 (2007) 880-886. https://doi.org/10.1110/ps.062712807.

[122] P. Angel, M. Karin, The role of Jun, Fos and the AP-1 complex in cell-proliferation and transformation, Biochim. Biophys. Acta - Rev. Cancer. 1072 (1991) 129-157. https://doi.org/10.1016/0304-419X(91)90011-9.

[123] T. Hai, F. Liu, E.A. Allegretto, M. Karin, M.R. Green, A family of immunologically related transcription factors that includes multiple forms of ATF and AP-1, Genes Dev. 2 (1988) 1216-1226. https://doi.org/10.1101/GAD.2.10.1216.

\section{Figures}




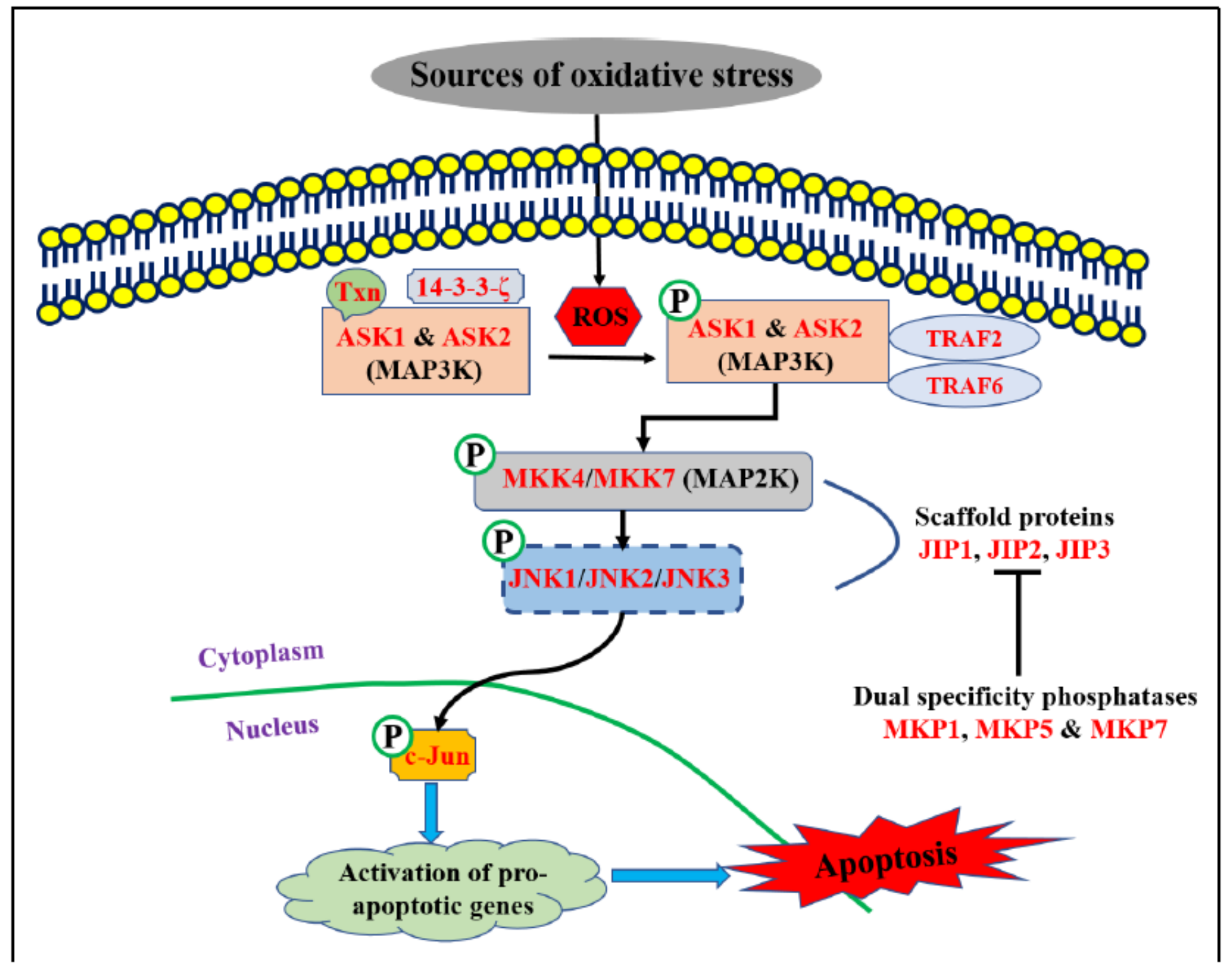

Figure 1

Schematic representation of oxidative stress-induced JNK signaling pathway. When a cell is subjected to oxidative stress, Trx dissociates from ASK1 followed by 14-3-3 Z. Free ASK1 either homodimerizes or forms a heterodimer with ASK2. This is followed by recruitment of TRAF2 and TRAF6 to ASK1 signalosome complex. ASK1 is activated either by trans-autophosphorylation (in homodimers) or is phosphorylated by ASK2 (in heterodimers). Active ASK1 triggers a cascade which results in phosphorylation of MAP2Ks, MKK4 and MKK7 followed by phosphorylation and activation of JNKs. The scaffold proteins (JIP1, JIP2 and JIP3) interact with all 3 tiers of MAPKs and potentiate JNK activation. Phosphorylated JNK translocate to the nucleus where it activates its substrate transcription factors such as c-Jun. It is through these substrates that JNK generates a stress response in the cell by altering gene expression. Upon removal of stress stimulus, it is important to inactivate JNKs and return cells to their normal state. This task is performed by dual specificity phosphatases (MKP1, MKP5 and MKP7). 


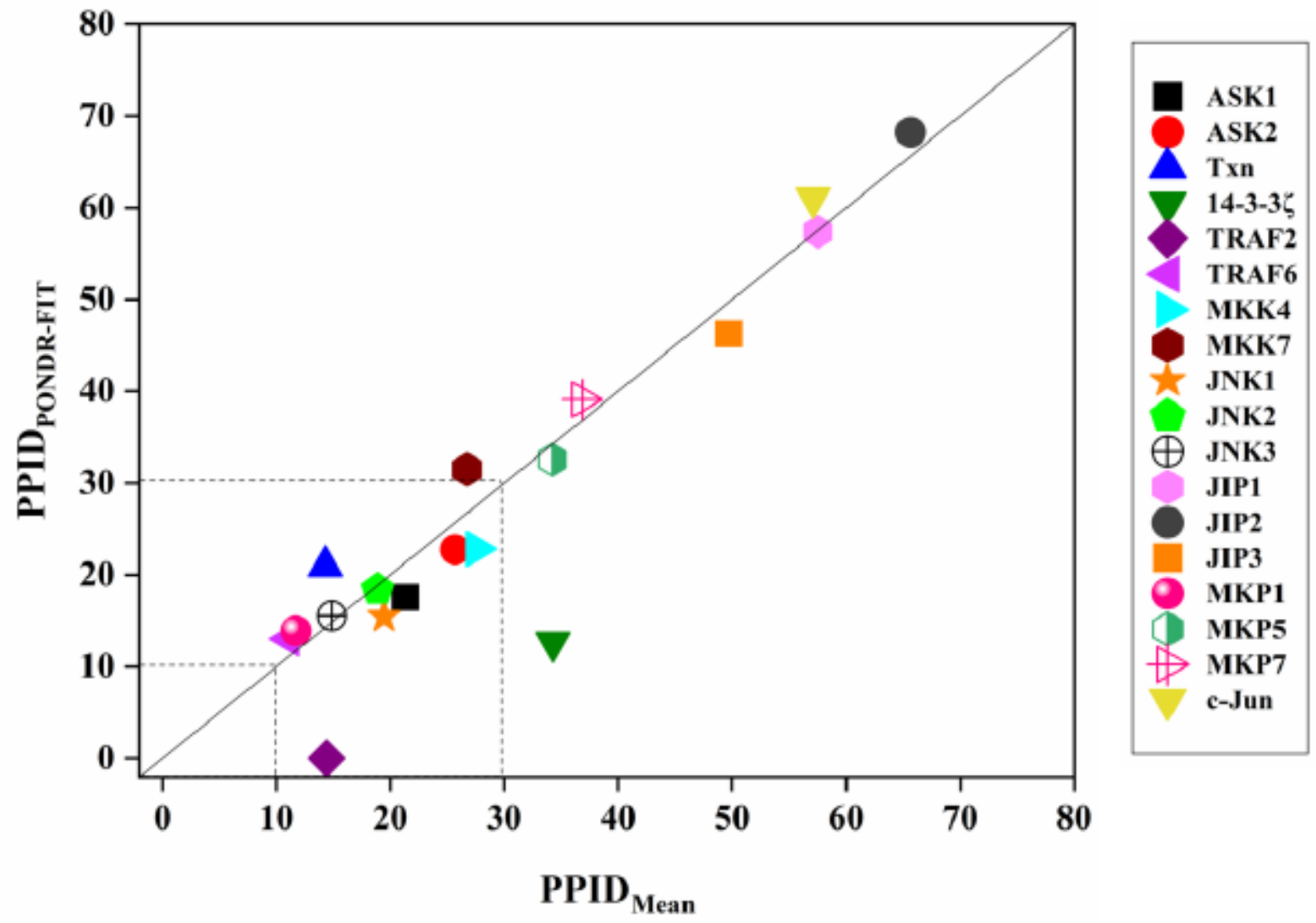

Figure 2

Evaluation of the disorder status of 18 proteins of the oxidative stress induced JNK signaling pathway: The 2D disorder plot represents the PPIDPONDR ${ }^{8} F I T$ ( $Y$ co-ordinate) vs. PPIDMean (X co-ordinate) dependence. 

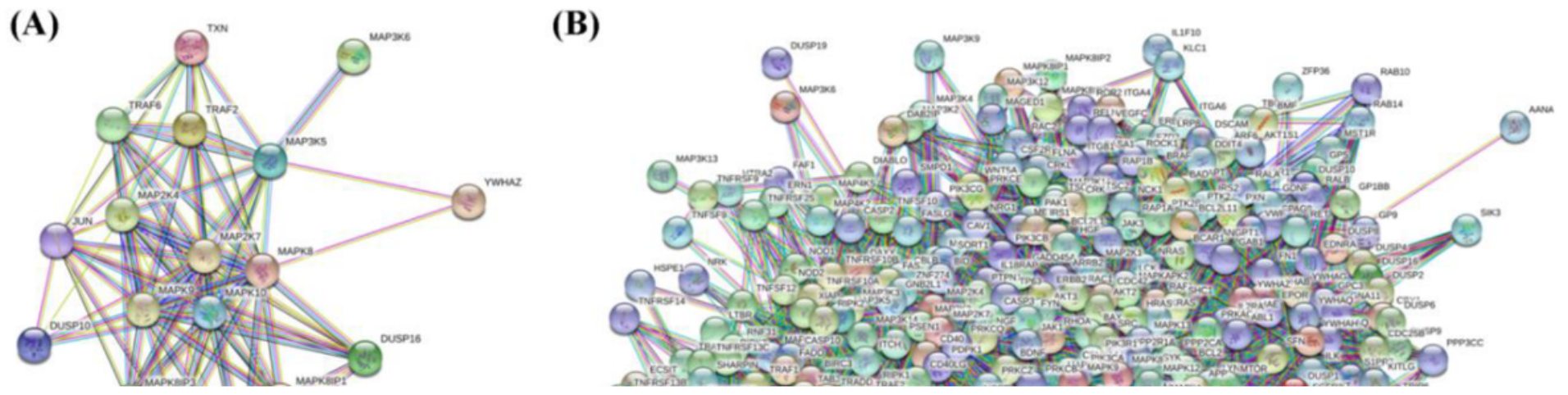

\section{Figure 3}

Evaluation of interactivity of proteins involved in the oxidative stress induced JNK signaling cascade. (A) Inter-set interactability of 18 proteins (ASK1/MAP3K5, ASK2/MAP3K6, Txn, 14-3-3ろ/YWHAZ, TRAF2, TRAF6, MKK4/MAP2K4, MKK7/MAP2K7, JNK1/MAPK8, JNK2/MAPK9, JNK3/MAPK10, JIP1/MAPK8IP1, JIP2/MAPK8IP2, JIP3/MAPK8IP3, MKP1/DSP1, MKP5/DSP10, MKP7/DSP16 and c-Jun/JUN) was analysed using STRING resource. A single PPI network including all proteins was generated using a medium confidence score of 0.4. (B) PPI network centred at the above mentioned 18 proteins of the JNK signaling cascade was obtained from STRING. This network includes 500 first shell interactions of the 18 query proteins and was generated using the highest confidence score of 0.9 . 
(A)

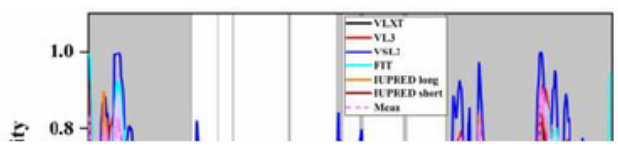

(B)

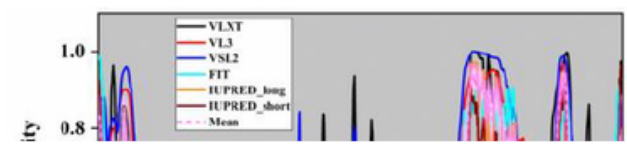

\section{Figure 4}

Intrinsic Disorder Analysis in ASK1 and ASK2: (A) Per-residue intrinsic disorder propensity of ASK1. (B) Intrinsic disorder propensity of ASK2. In plots (A) and (B), disorder profiles of ASK1 and ASK2 were generated by PONDR ${ }^{\circledR}$ VLXT (black line), PONDR® VL3 (red line), PONDR® VSL2 (blue line), PONDR® FIT (cyan line), IUPRED long (orange line), and IUPRED short (wine line). The magenta dotted line represents the mean disorder propensity calculated using the disorder scores from all six predictors and 
the magenta shadow region represents the mean error distribution. The grey shaded region in plot (A) represents the residues of the protein for which either no PDB structure is available or the residues which are missing in the available PDB structures. (A1) $2.10 \AA$ resolution structure of the central regulatory region (residues 269 - 658) in ASK1 homodimer (PDB ID: 5ULM). (A2) 2.11 Å resolution, structure of residues 660 - 977 of $A S K 1$, including the kinase domain (PDB ID: 4BF2). The protein structures in (A1) and (B1) have been shown in green colour. As no PDB structure for ASK2 is available, the entire plot (B) is shaded grey. (A3) (B1) Evaluation of intrinsic disorder, MoRF regions and PTM sites in ASK1 and ASK2 by D2P2.

(A)

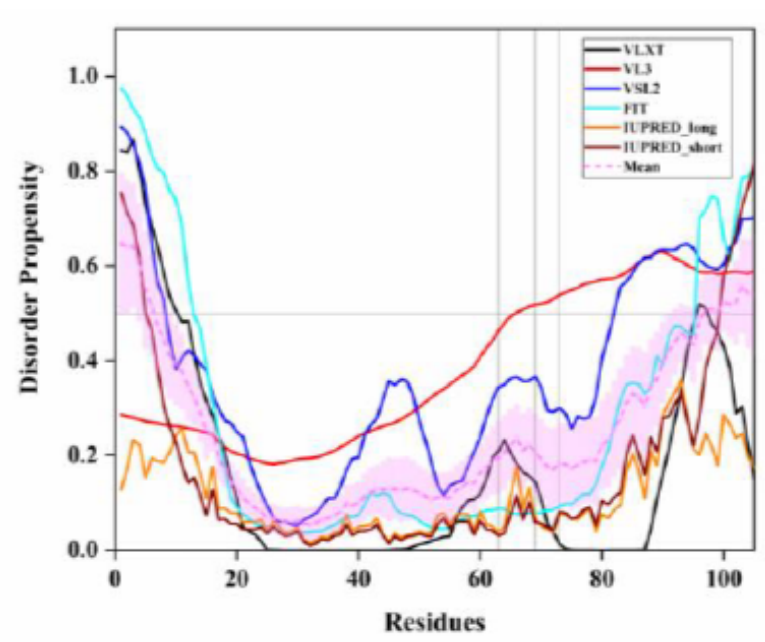

(A1)

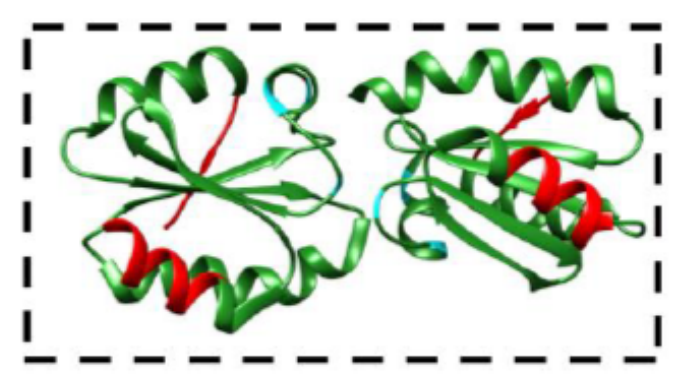

(A2)

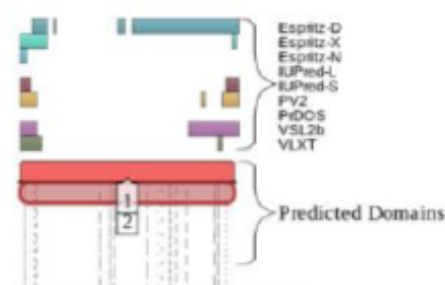

Predicted Disorder Agreement

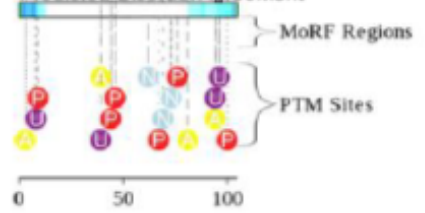

Key:

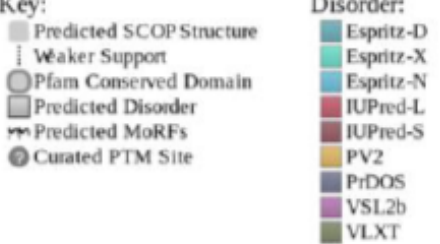

(B)

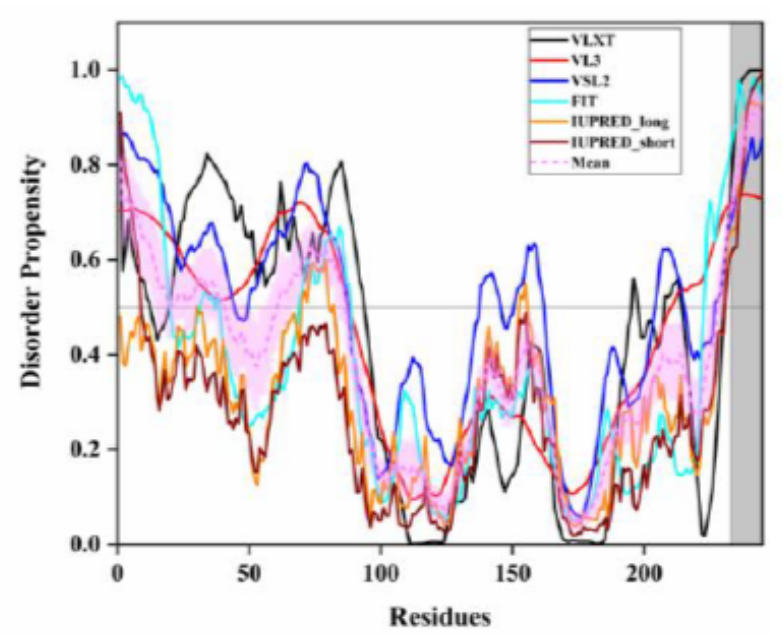

(B1)

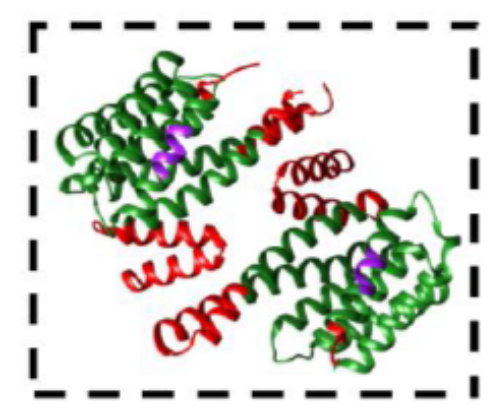

(B2)

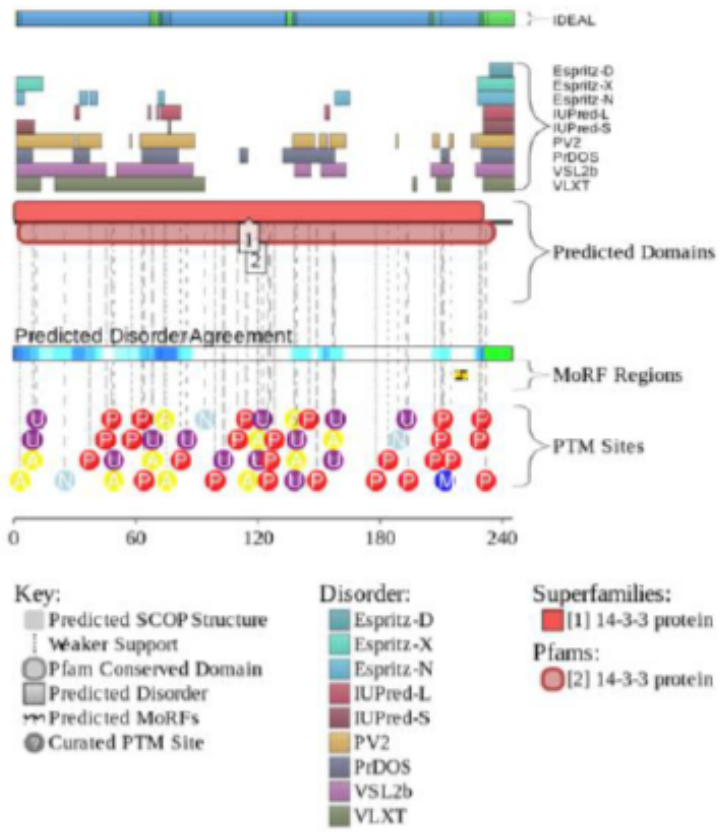




\section{Figure 5}

Intrinsic Disorder Analysis in Txn and 14-3-3ろ: (A) Disorder Propensity of residues in Txn. (B) Disorder Propensity of residues in 14-3-3ろ. In plots (A) and (B), disorder profiles of Txn and 14-3-3 3 generated by PONDR® VLXT (black line), PONDR® VL3 (red line), PONDR® VSL2 (blue line), PONDR® FIT (cyan line), IUPRED long (orange line) and IUPRED short (wine line) have been represented. The magenta dotted line represents the mean disorder propensity calculated using the disorder scores from all six predictors and the magenta shadow region represents the mean error distribution. The grey shaded vertical lines in plot (A) represents the residues which are mutated in the available PDB structure, while, the grey shaded region in plot (B) represents the residues of the protein for which either no PDB structure is available or the residues which are missing in the available PDB structure. (A1) $0.97 \AA$ resolution full length structure of Txn homodimer with three mutated residues. (PDB ID: 4004). (B1) $2.00 \AA$ resolution, full length structure of 14-3-3 3 homodimer (PDB ID: 1QJB). The protein structures in (A1) and (B1) have been shown in green colour. The protein residues which fall in the IDPRs have been coloured red, the MoRF regions have been shown in purple and the mutated residues in Txn have been represented in cyan colour in PDB structures. (A2) (B2) Evaluation of intrinsic disorder, MoRF regions and PTM sites in Txn and 14-3-3Z by D2P2. 
(A)

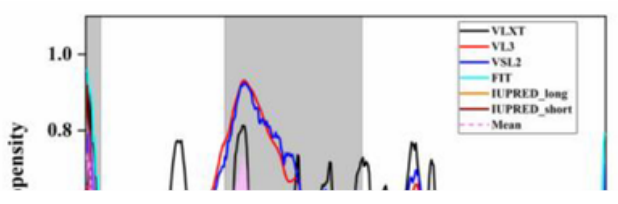

(B)

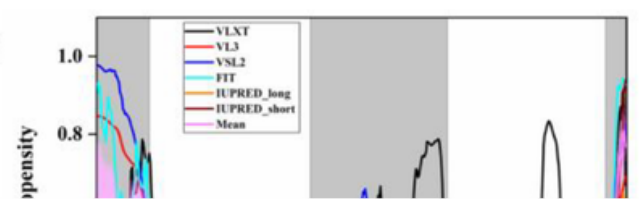

\section{Figure 6}

Intrinsic Disorder Analysis and structural characterization of TRAF2 and TRAF6: (A) Per-residue intrinsic disorder propensity of TRAF2. (B) Disorder propensity of TRAF6.In plots (A) and (B), disorder profiles of TRAF2 and TRAF6 generated by PONDR® VLXT (black line), PONDR® VL3 (red line), PONDR® VSL2 (blue line), PONDR® FIT(cyan line), IUPRED long (orange line)and IUPRED short (wine line) have been represented. The magenta dotted line represents the mean disorder propensity calculated using the disorder scores from all six predictors and the magenta shadow region represents the mean error distribution. The grey shaded region in the graph represents the residues of the protein for which either no PDB structure is available or the residues which are missing in the available PDB structures. (A1) $1.90 \AA$ resolution structure of the RING domain and first zinger finger domain of TRAF2 (PDB ID: 3KNV). (A2) $2.61 \AA$ resolution structure of the residues $266-328$ in TRAF2 homotrimer (PDB ID: 3MOA). (A3) $2.4 \AA$ resolution structure of the TRAF domain in TRAF2 homotrimer (PDB ID: 1QSC). (B1) $2.20 \AA$ resolution structure of the N-terminal domain of TRAF6 homodimer (PDB ID: 3HCS). (B2) $1.80 \AA$ A resolution structure of the TRAF domain in TRAF6 (PDB ID: 1LB6). The protein structures in (A1), (A2), (A3), (B1) and (B2) 
have been shown in green colour. The protein residues which fall in the IDPRs have been coloured red in these structures. (A4) (B3) Evaluation of Intrinsic disorder, MoRF regions and PTM sites in TRAF2 and TRAF6 by D2P2.

(A)

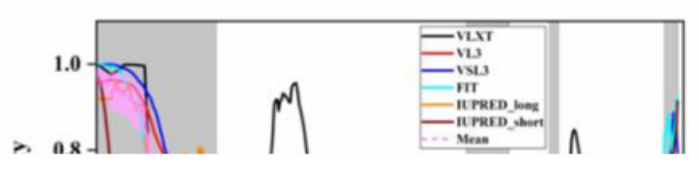

(B)

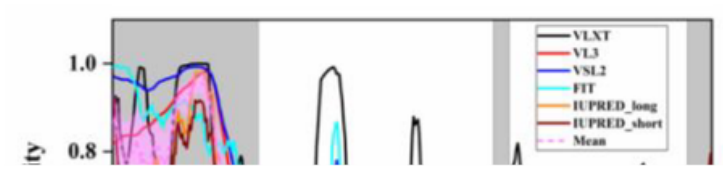

\section{Figure 7}

Intrinsic disorder analysis of MKK4 and MKK7: (A) Per-residue disorder predisposition of MKK4. (B) Disorder propensity of MKK7. In plots (A) and (B), disorder profiles of MKK4 and MKK7 generated by PONDR® VLXT (black line), PONDR® VL3 (red line), PONDR® VSL2 (blue line), PONDR® FIT (cyan line), IUPRED long (orange line) and IUPRED short (wine line) have been represented. The magenta dotted line represents the mean disorder propensity calculated using the disorder scores from all six predictors and the magenta shadow region represents the mean error distribution. The grey shaded region in the graph 
represents the residues of the protein for which either no PDB structure is available or the residues which are missing in the available PDB structures. (A1) $2.30 \AA$ resolution structure containing the protein kinase domain and DVD domain of MKK4 homotrimer (PDB ID: 3ALN). (B1) $2.10 \AA$ resolution structure of the protein kinase domain and DVD domain of MKK7 (PDB ID: 5B2L). The protein structures in (A1) and (B1) have been shown in green colour. The protein residues which fall in the IDPRs have been coloured red, the MoRF regions have been shown in purple and the IDP residue (Residue: 83) in chain A of MKK4 homotrimer which also lies in the MoRF region is represented in cyan colour PDB structures. (A2) (B2) Evaluation of intrinsic disorder, MoRF regions and PTM sites in MKK4 and MKK7 by D2P2.

(A)

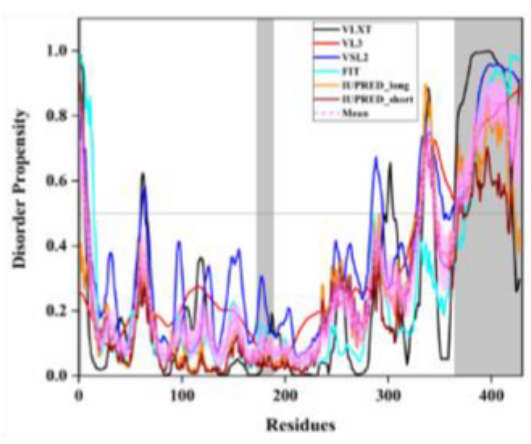

(A1)

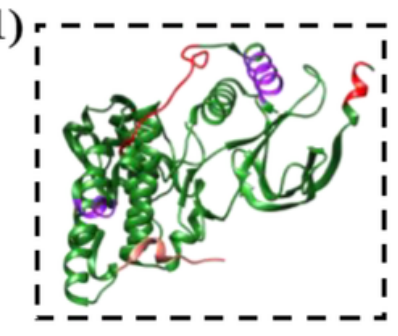

(A2)

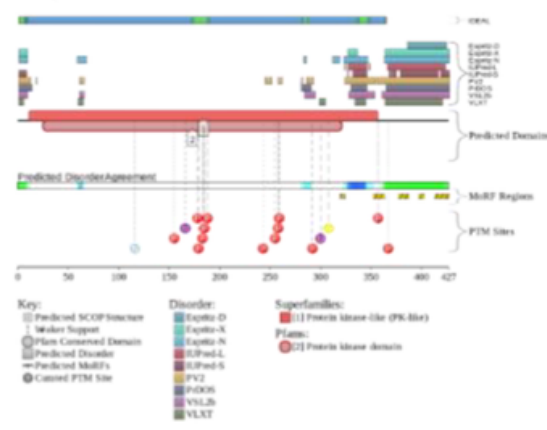

(B)

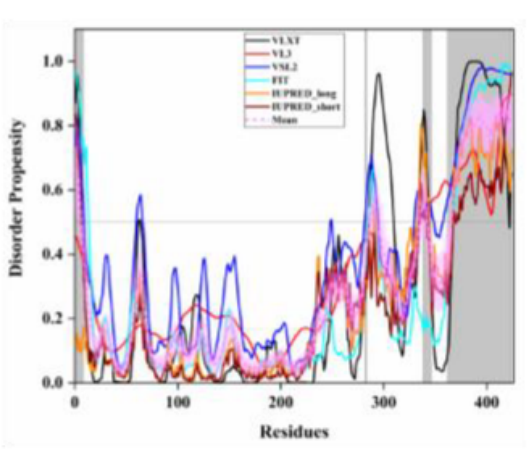

(C)

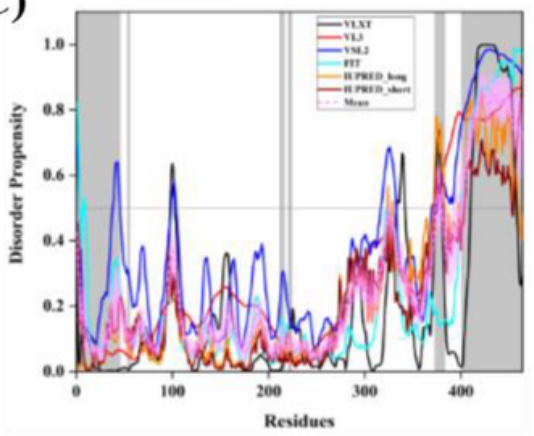

(B1)

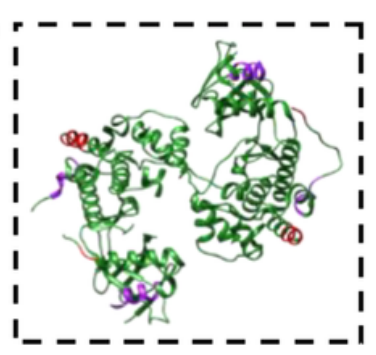

(B2)

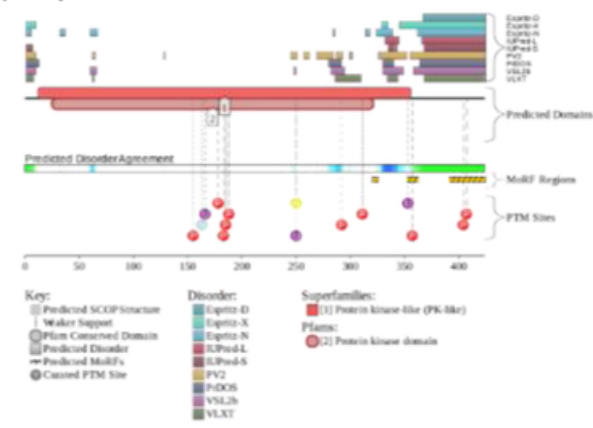

(C1)

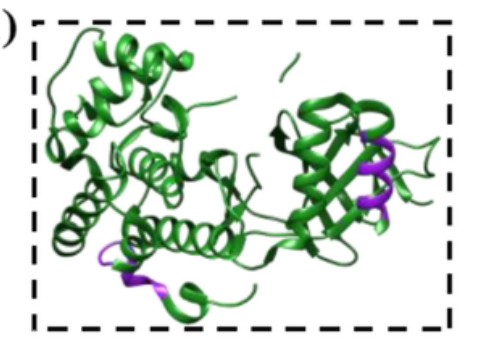

(C2)

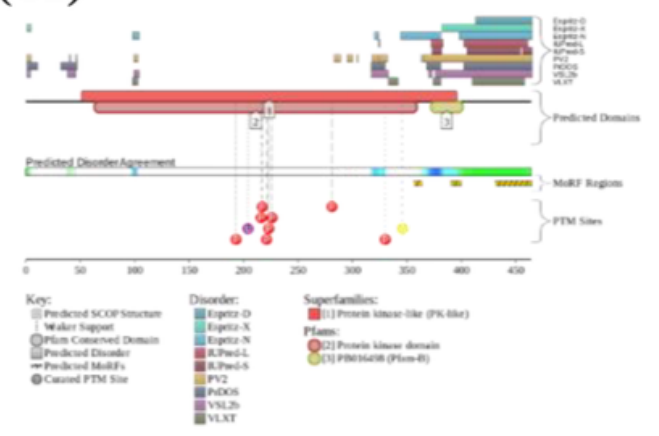

Figure 8

Intrinsic disorder analysis in JNK1, JNK2, and JNK3: (A) Disorder propensity of JNK1. (B) Disorder predisposition of JNK2. (C) Per-residue disorder profile of JNK3. In plots (A), (B) and (C), disorder profiles of JNK1, JNK2 and JNK3 generated by PONDR® VLXT (black line), PONDR® VL3 (red line), PONDR® VSL2 (blue line), PONDR® FIT(cyan line), IUPRED long (orange line) and IUPRED short (wine line) are shown. The magenta dotted line represents the mean disorder propensity calculated using the disorder scores from all six predictors and the magenta shadow region represents the mean error distribution. The grey shaded region in the graph represents the residues of the protein for which either no PDB structure is available or the residues which are missing in the available PDB structures. (A1) $1.33 \AA$ resolution 
structure of residues 2 - 364 containing the protein kinase domain and TxY activation motif of JNK1 in complex with a short peptide from the Nuclear factor of activated T-cells, cytoplasmic 3 (NFATC3) (PDB ID: 2XRW). (B1) $2.14 \AA$ resolution structure of residues 1 - 361 containing the protein kinase domain and the TxY activation motif of JNK2 homodimer (PDB ID: 3E70). (C1) $2.10 \AA$ resolution structure of the fulllength JNK3 (PDB ID: 3TTJ). The JNK1, JNK2 and JNK3 protein structures in (A1), (B1) and (C1) have been shown in green colour. The protein residues which fall in the IDPRs have been coloured red, the MoRF regions have been shown in purple and the NFATC3 protein which is bound to JNK1 in (A1) has been coloured salmon pink. (A2) (B2) (C2) Evaluation of intrinsic disorder, MoRF regions and PTM sites in JNK1, JNK2 and JNK3 by D2P2.
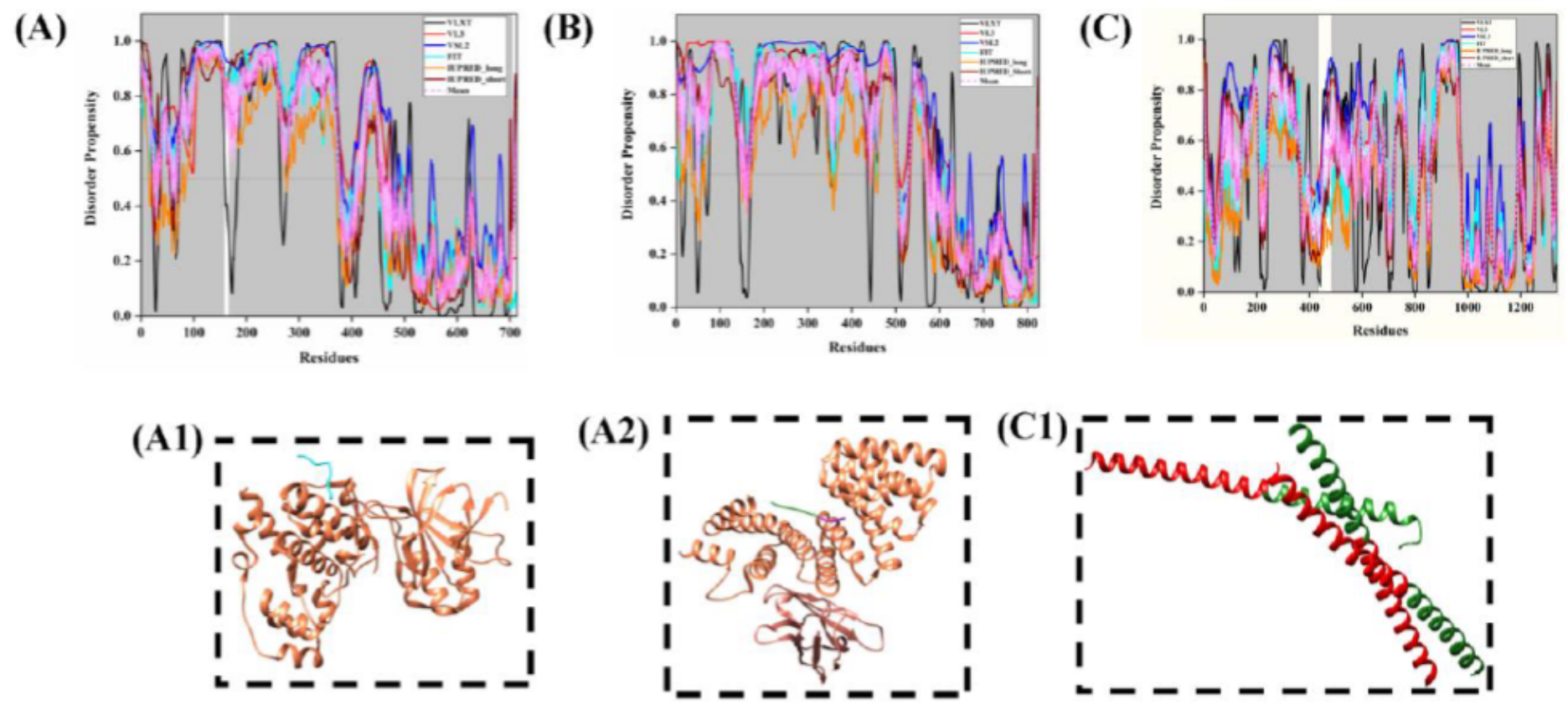

(A3)

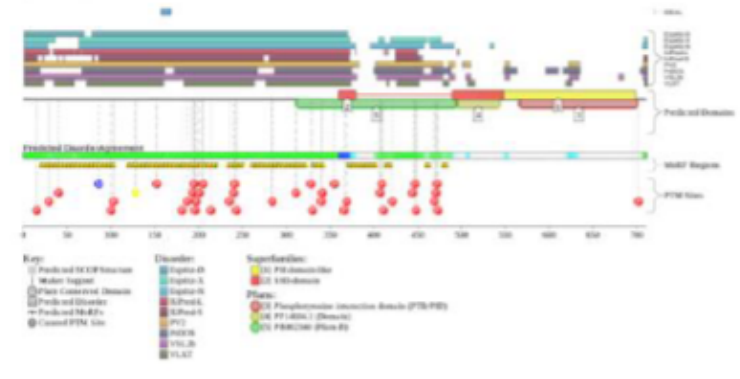

(C2)

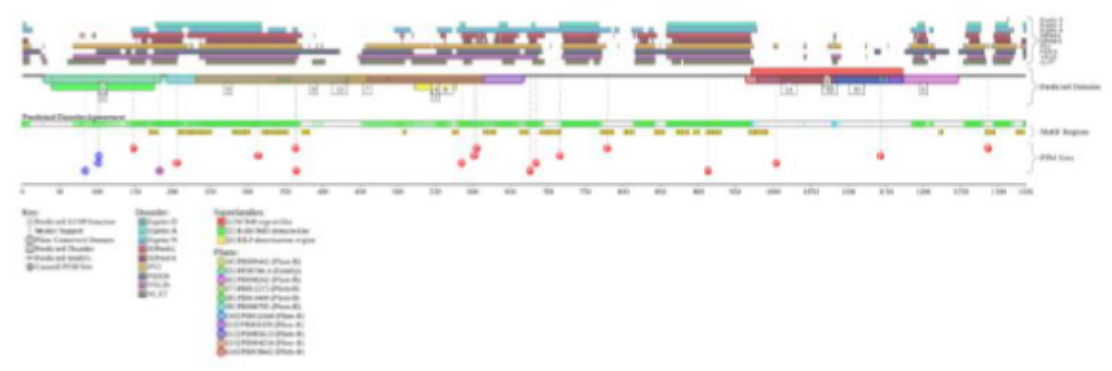

\section{Figure 9}

Intrinsic Disorder Analysis in JIP1, JIP2 and JIP3:(A) Disorder propensity of JIP1. (B) Disorder predisposition of JIP2. (C) Per-residues disorder profile of JIP3. In plots (A), (B) and (C), disorder profiles of JIP1, JIP2 and JIP3 generated by PONDR® VLXT (black line),PONDR® VL3 (red line), PONDR® VSL2 (blue line), PONDR ${ }^{\circledR}$ FIT(cyan line), IUPRED long (orange line) and IUPRED short (wine line) have been represented. The magenta dotted line represents the mean disorder propensity calculated using the disorder scores from all six predictors and the magenta shadow region represents the mean error distribution. The grey shaded region in the graph represents the residues of the protein for which either no 
PDB structure is available or the residues which are missing in the available PDB structures. (A1) $1.99 \AA$ resolution structure of the residues 158 - 167 of JIP1 in complex with JNK3 (PDB ID: 4H39). (A2) $2.70 \AA$ resolution structure of residues $704-711$ of JIP1 fused with TPR domain of KLC1 in complex with a nanobody (PDB ID: 6FUZ).(C1) $2.06 \AA$ resolution structure of the leucine zipper II domain in JIP3 homotrimer (PDB ID: 4PXJ). The JIP1 and JIP3 protein structures in (A1), (A2) and (C1) have been shown in green colour. The proteins JNK3 and nanobody, which are bound to JIP1 peptides in (A1) and (A2) have been indicated in coral colour, and the protein KLC1, which is fused to JIP1 peptide in (A2) has been shown in salmon pink. The protein residues, which fall in the IDPRs have been coloured red, and the IDPRs which fall into MoRF regions have been shown in cyan colour. (A3) (B1) (C2) Evaluation of intrinsic disorder, MoRF regions and PTM sites in JIP1, JIP2 and JIP3 by D2P2.

(A)

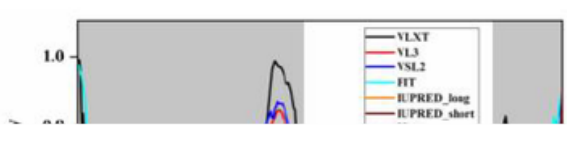

(B)

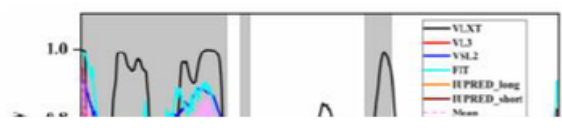

(C)

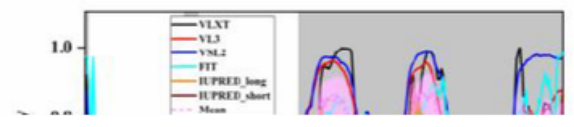

\section{Figure 10}

Intrinsic Disorder Analysis in MKP1, MKP5 and MKP7: (A) Disorder Propensity of residues in MKP1. (B) Disorder Propensity of residues in MKP5. (C) Disorder Propensity of residues in MKP7. In plots (A), (B) and (C), disorder profiles of MKP1, MKP5 and MKP7 generated by PONDR® VLXT (black line), PONDR® VL3 (red line), PONDR® VSL2 (blue line), PONDR® FIT(cyan line), IUPRED long (orange line) and IUPRED short (wine line) have been represented. The magenta dotted line represents the mean disorder propensity 
calculated using the disorder scores from all six predictors and the magenta shadow region represents the mean error distribution. The grey shaded region in the graph represents the residues of the protein for which either no PDB structure is available or the residues which are missing in the available PDB structures. (A1) 2.23A四 resolution structure of catalytic domain of MKP1 (green colour) fused with MBP protein (coral colour) and in complex with AR protein (salmon pink). (PDB ID: 6D66). The histidine tag has been shown in yellow colour. (B1) 2.20 ARQ resolution structure of MAPK binding domain (green colour) (PDB ID: 2OUC). (B2) $2.80 \mathrm{~A}$ 㽖 resolution structure of the catalytic domain of MKP5 (green colour)

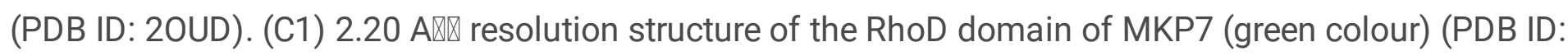
2VSW). (C2) Structure of the residues $156-301$ of MKP7 (green colour) in complex with JNK1 (Salmon pink) (PDB ID: 4YR8). In the protein structures, the residues which fall in the IDPRs have been coloured red and the MoRF regions have been shown in purple colour. (A2) (B3) (C3) Evaluation of intrinsic disorder, MoRF regions and PTM sites in MKP1, MKP5 and MKP7 by D2P2. 
(A)

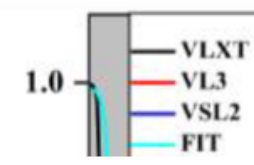

(A1)

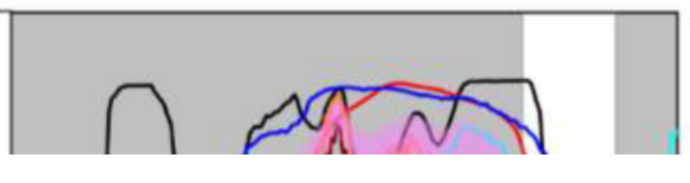

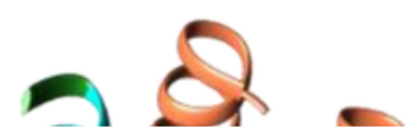

\section{Figure 11}

Intrinsic Disorder Analysis in c - Jun: (A) Disorder propensity of c - Jun. In plot (A), disorder profile of c Jun generated by PONDR® VLXT (black line), PONDR® VL3 (red line), PONDR® VSL2 (blue line), PONDR ${ }^{\circledR}$ FIT (cyan line), IUPRED long (orange line) and IUPRED short (wine line) have been represented. The magenta dotted line represents the mean disorder propensity calculated using the disorder scores from all six predictors and the magenta shadow region represents the mean error distribution. The grey 
shaded region in the graph represents the residues of the protein for which either no PDB structure is available or the residues which are missing in the available PDB structure. (A1) $1.99 \AA$ resolution structure of the residues 277 - 308 of $c$ - Jun in complex with FOSW (PDB ID: 5FV8). The c - Jun protein structure in (A1) has been shown in green colour. The protein FOSW, which is bound to $c$ - Jun peptide in (A1) has been indicated in coral colour. The protein residues, which fall in the IDPRs have been coloured red, and the IDPRs which fall into MoRF regions have been shown in cyan colour. (A2) Evaluation of intrinsic disorder, MoRF regions and PTM sites in c - Jun by D2P2.

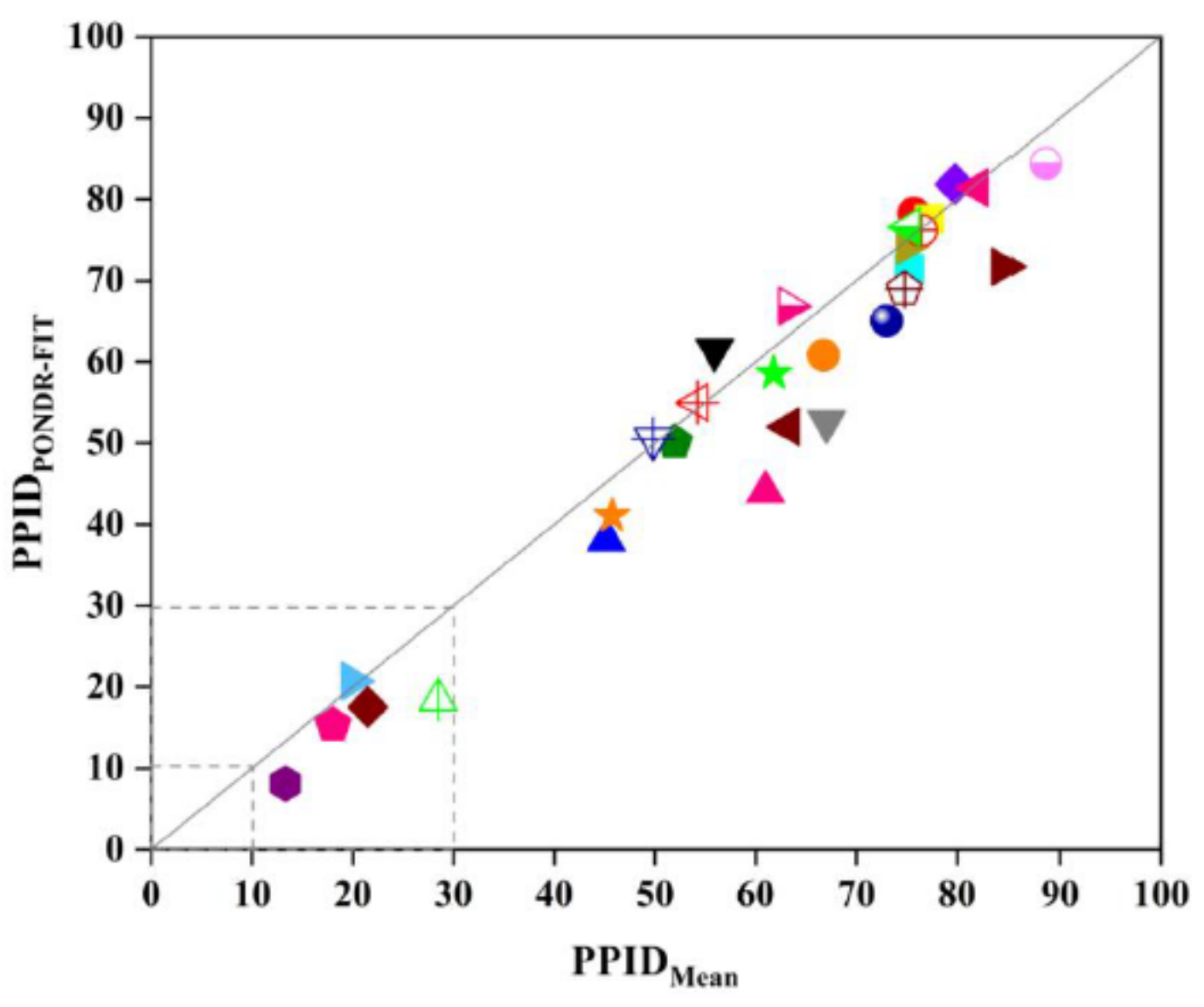

\begin{tabular}{|c|}
\hline $\begin{array}{l}\text { ATF2 } \\
\text { Elk1 } \\
\text { Elk3 } \\
\text { Elk4 } \\
\text { FOXO3 } \\
\text { FOXO4 } \\
\text { Gli1 } \\
\text { Gli3 } \\
\text { Hes1 } \\
\text { HSF1 } \\
\text { JDP2 } \\
\text { JunD } \\
\text { Myc } \\
\text { NFAT1 } \\
\text { NFAT2 } \\
\text { NFAT4 } \\
\text { NF-E2 } \\
\text { Nr1 } \\
\text { p53 } \\
\text { p73 } \\
\text { RRN3 } \\
\text { Smad2 } \\
\text { Smad3 } \\
\text { Sp1 } \\
\text { STAT1 } \\
\text { STAT3 } \\
\text { Twist1 } \\
\text { POU6F1 } \\
\text { Runx2 }\end{array}$ \\
\hline
\end{tabular}

\section{Figure 12}

Evaluation of the disorder status of 29 Transcription factors which are known substrates of JNK: The 2D disorder plot represents the PPIDPONDR®FIT (Y co-ordinate) vs. PPIDMean (X co-ordinate) dependence.

\section{Supplementary Files}

This is a list of supplementary files associated with this preprint. Click to download.

- SupplementaryMaterials.docx 